\title{
MULTISTATIONARITY IN SEQUENTIAL DISTRIBUTED MULTISITE PHOSPHORYLATION NETWORKS
}

\author{
KATHARINA HOLSTEIN, DIETRICH FLOCKERZI AND CARSTEN CONRADI
}

\begin{abstract}
Multisite phosphorylation networks are encountered in many intracellular processes like signal transduction, cell-cycle control or nuclear signal integration. In this contribution networks describing the phosphorylation and dephosphorylation of a protein at $n$ sites in a sequential distributive mechanism are considered. Multistationarity (i.e. the existence of at least two positive steady state solutions of the associated polynomial dynamical system) has been analyzed and established in several contributions. It is, for example, known that there exist values for the rate constants where multistationarity occurs. However, nothing else is known about these rate constants.

Here we present a sign condition that is necessary and sufficient for multistationarity in $n$ site sequential, distributive phosphorylation. We express this sign condition in terms of linear systems and show that solutions of these systems define rate constants where multistationarity is possible. We then present, for $n \geq 2$, a collection of feasible linear systems and hence give a new and independent proof that multistationarity is possible for $n \geq 2$. Moreover, our results allow to explicitly obtain values for the rate constants where multistationarity is possible. Hence we believe that, for the first time, a systematic exploration of the region in parameter space where multistationarity occurs has become possible. One consequence of our work is that, for any pair of steady states, the ratio of the steady state concentrations of kinase-substrate complexes equals that of phosphatase-substrate complexes.
\end{abstract}

Keywords: sequential distributed phosphorylation; mass-action kinetics; multistationarity; sign condition; rate constants

\section{INTRODUCTION}

Protein phosphorylation and dephosphorylation is frequently encountered in procaryotic and in eukaryotic cells. Thereby the majority of proteins is phosphorylated at more than one phosphorylation site (with most procaryotic proteins having $n \leq 7$ phosphorylation sites and eukaryotic proteins generally having a large number of phosphorylation sites - certain eukaryotic proteins have up to $n=150$ sites [26]). Multisite phosphorylation networks are consequently encountered in many intracellular processes, including, among others, the following: (i) signal transduction networks from the family of Mitogen Activated Kinases (MAPK cascades), where the core module consists of one protein phosphorylated at a single site and two proteins each phosphorylated at two sites (see e.g. [16, 23, 24, 31]); (ii) check-points in cell-cycle control, for example the control of the G1/S transition in budding yeast involving the protein Sic1 with $n=9$ (see e.g. [1, 2, 28]) and (iii) nuclear signal integration, for example, in mammalian cells involving members of the NFAT transcription factor family with $n=13$ (see e.g. [7, 8, 14, 18, 27]).

In this contribution we focus on networks describing the phosphorylation and dephosphorylation of a single protein $A$ at $n$ sites in a sequential distributive mechanism (cf. Figure 1 and, for example, [13]). Such a mechanism is able to cover the essential aspects of proteins like the aforementioned Sic1 or the members of the NFAT family [21, 22].

Due to the biological significance of sequential distributive phosphorylation, mathematical models have been studied in a variety of publications. Several publications deal with double phosphorylation (i.e. $n=2$ ) and establish multistationarity [4, 5, 6, 11] (i.e. the existence of at least two positive steady state solutions, cf. Definition 3.5) and bistability [19] (i.e. the existence of two (asymptotically) stable steady state solutions). Networks with $n$ phosphorylation sites have been studied, for 


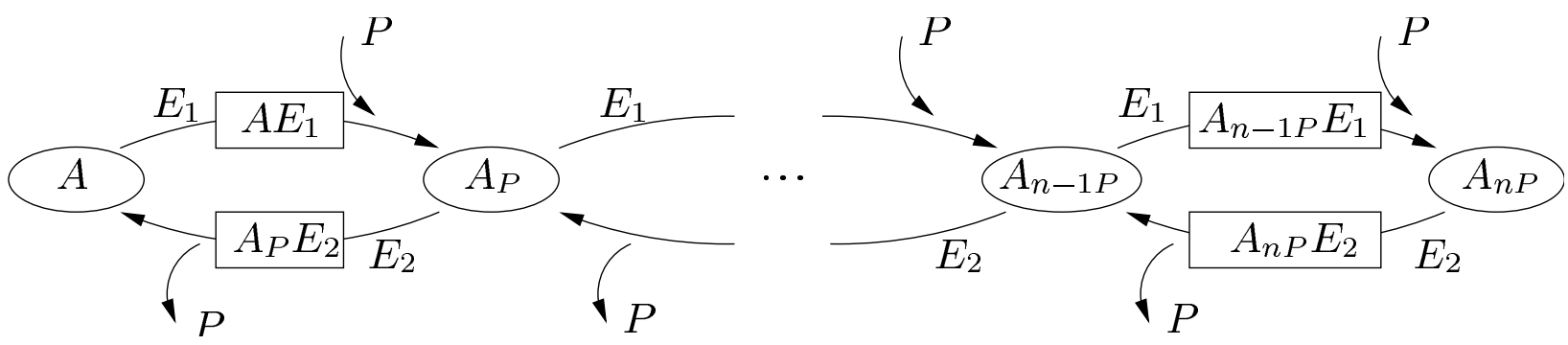

FiguRE 1. Network describing the sequential distributive phosphorylation and dephosphorylation of protein $A$ at $n$-sites by a kinase $E_{1}$ a phosphatase $E_{2}$. The phosphorylated forms of $A$ are denoted by the subscript $n P$ (denoting the number of phosphorylated sites).

example, in [21, 22], where bistability is demonstrated numerically and in [12], where it is argued that even though bistability is in principle possible, it is unlikely to occur in-vivo.

The set of positive steady states of networks with $n$ phosphorylation sites has been studied algebraically in [25] and in [20]. In [25] the set of positive steady states of the more general class of so called post translational modification networks (with $n$-site sequential distributive phosphorylation as a special case) is analyzed. Such networks admit a rational parameterization of the set of positive steady states. And in [20] it is shown that for $n$-site sequential distributive phosphorylation this set is defined by a binomial ideal. Hence $n$-site sequential distributive phosphorylation is an instance of a Chemical Reaction System with toric steady states. In [20] a condition for multistationarity in these reaction systems is given in terms of a sign condition on elements of two linear subspaces. Based on the results presented in [3, 4], this condition can be restated in terms of linear systems: to decide the sign condition, a large set of linear systems has to be examined and multistationarity is possible, if and only if at least one of these is feasible (cf. [20, Theorem 5.5] and [3, Lemma 2 \& Theorem 2]).

The number of steady states in general $n$-site sequential distributive phosphorylation has been studied in [30]. There upper and lower bounds have been established: there exist parameter values such that, for $n$ even (odd), there are at least $n+1(n)$ steady states. And for all (positive) parameter values there are at most $2 n-1$ steady states. Hence multistationarity has been established for sequential distributive phosphorylation in [30]. However, no information other than existence is given about the parameter values where multistationarity is possible. In this contribution we address this question: we present for every $n \geq 2$ a collection of feasible linear systems and show that every solution of one of those systems defines parameter values where multistationarity is possible (together with two positive steady states as witness). Thus these results not only constitute a new and independent proof that multistationarity is possible for $n \geq 2$, they additionally enable, for the first time, a systematic exploration of the region in parameter space where multistationarity is possible. Due to the ubiquity of multisite phosphorylation this may be of potential interest to researchers working in many fields of (quantitative) biology. For the purpose of finding parameter values in biologically meaningful ranges we will start with this exploration in [15].

We arrive at our main result on the basis of our previous work from [3, 4] and [20]: as in the aforementioned references we derive a sign condition that is necessary and sufficient for the existence of multistationarity (Theorem $4.2 \& 4.6$ ). In contrast to [3, 20] our condition is formulated in terms of the sign patterns generated by two linear subspaces (given as the image of two matrices defined in eqns. (8e) \& (17c)). We then exploit the fact that every sign pattern uniquely defines a linear system to restate the sign condition in terms of these linear systems: multistationarity is possible, if and only if at least one out of $\frac{1}{2}\left(3^{(3 n+3)}-1\right)$ or $2^{(3 n+2)}$ linear systems is feasible depending on whether some or all components of the two witness steady state vectors have to differ to qualify 
for multistationarity, cf. Definition 3.5 and Remark 4.7. For the latter case, we present formulae to construct all sign patterns defining feasible linear systems (cf. Theorem 4.10) and show that every solution of one of these linear systems defines parameter values where multistationarity occurs with two positive steady states differing in every component. We show that there are $2(n-1)(n+2)$ such sign patterns (cf. Proposition 4.14).

This paper is organized as follows: Section 2 introduces some of the basic notations. In Section 3 a mass action network is derived from Fig. 1 and the associated dynamical system is defined along with a formal definition of multistationarity. In Section 4 the main results address multistationarity in terms of sign patterns defining feasible linear systems (cf. Theorem $4.2 \& 4.10$ ). The proofs of two of these results have been relegated to separate Sections $5 \& 6$ for easier reading.

\section{Notation}

We use the symbol $\mathbb{R}^{m}$ to denote Euclidian $m$-space, the symbol $\mathbb{R}_{\geq 0}^{m}$ to denote the nonnegative orthant and $\mathbb{R}_{>0}^{m}$ to denote the interior of the nonnegative orthant. Vectors are considered as column vectors and, for convenience, usually displayed as row vectors using ${ }^{T}$ to denote the transpose. For example, $x \in \mathbb{R}^{m}$ will usually be displayed as $\left(x_{1}, \ldots, x_{m}\right)^{T}$.

For positive vectors $x \in \mathbb{R}_{>0}^{m}$ we use the shorthand notation $\ln x$ to denote

$$
\ln x:=\left(\ln x_{1}, \ldots, \ln x_{m}\right)^{T} \in \mathbb{R}^{m} .
$$

Similarly, for $x \in \mathbb{R}^{m}$, we use $e^{x}$ to denote

$$
e^{x}:=\left(e^{x_{1}}, \ldots, e^{x_{m}}\right)^{T} \in \mathbb{R}_{>0}^{m}
$$

and, for $x \in \mathbb{R}^{m}$ with $x_{i} \neq 0, i=1, \ldots, m$,

$$
x^{-1}:=\left(\frac{1}{x_{1}}, \ldots, \frac{1}{x_{m}}\right)^{T} \in \mathbb{R}^{m} .
$$

Finally, $x^{y}$ with $x, y \in \mathbb{R}_{\geq 0}^{m}$ will be defined by

$$
x^{y}:=\prod_{i=1}^{m} x_{i}^{y_{i}} \in \mathbb{R}_{\geq 0}^{m}
$$

The set of real $(p \times q)$-matrices with $p$ rows and $q$ columns will be denoted by $\mathbb{R}^{p \times q}$. For $p$ dimensional column vectors $s_{(j)}=\left(s_{1 j}, \ldots, s_{p j}\right)^{T} \in \mathbb{R}^{p}, j=0,1, \ldots, q$, we denote the $p(q+1)$ dimensional column vector $\left(s_{10}, \ldots, s_{p 0}, s_{11}, \ldots, s_{p 1}, \ldots \ldots, s_{1 q}, \ldots, s_{p q}\right)^{T}$ by

$$
\operatorname{col}\left(s_{(0)}, \ldots, s_{(q)}\right) \in \mathbb{R}^{p(q+1)} \text { with the matrix }\left(s_{(0)}, \ldots s_{(q)}\right) \in \mathbb{R}^{p \times(q+1)} .
$$

\section{Mathematical MOdels of $n$-Site SEQUEnTial Distributive PHOSPHORYLATION}

Here we first introduce the notation used to describe dynamical systems defined by mass action networks by means of the example network depicted in Fig. 2. Then we discuss the mass action network derived from Fig.1 and present the dynamical system defined by this mass action network. 


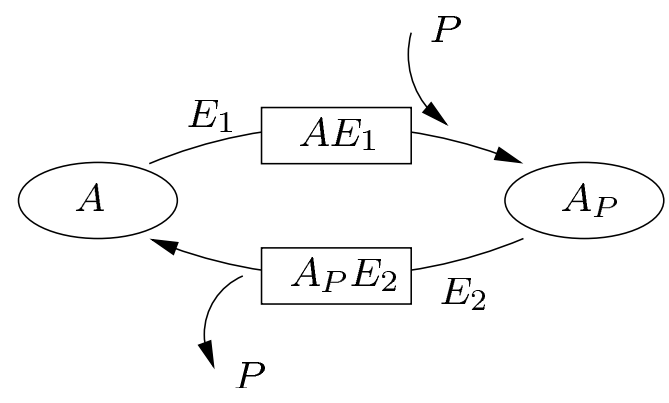

(A) 1-site phosphorylation

$$
\begin{aligned}
& \stackrel{x_{1}}{E_{1}}+\stackrel{x_{2}}{A \stackrel{k_{1}}{\rightleftharpoons}} \underset{k_{2}}{\rightleftharpoons} A \stackrel{x}{E}_{1} \stackrel{k_{3}}{\longrightarrow} \stackrel{x_{1}}{E_{1}}+\stackrel{x_{5}}{A_{P}} \\
& \stackrel{x_{3}}{E_{2}}+\stackrel{x_{5}}{A_{P}} \underset{l_{2}}{\stackrel{l_{1}}{\rightleftharpoons}} A_{P}^{x_{6}} E_{2} \stackrel{l_{3}}{\longrightarrow} \stackrel{x_{3}}{E_{2}}+\stackrel{x_{2}}{A}
\end{aligned}
$$

(в) Mass action network

Figure 2. Phosphorylation of protein $A$ at a single phosphorylation site: process diagram (A) and mass action network (B).

3.1. Dynamical systems defined by a single phosphorylation network. The network depicted in Fig. 2 describes the phosphorylation of a protein $A$ at a single site by the kinase $E_{1}$ and the dephosphorylation of mono-phosphorylated protein $A_{P}$ by the phosphatase $E_{2}$ (cf. Fig. 2a). Figure $2 \mathrm{~b}$ contains a mass action network describing this process, where chemical reactions are denoted by arrows pointing from the species that are consumed in a reaction (the educts) to the species that are produced (the products) and $k_{i}\left(l_{i}\right)$ denote rate constants. The network consists of 6 species, the kinase $E_{1}$ (with concentration variable $x_{1}$ ), the protein $A\left(x_{2}\right)$, the phosphatase $E_{2}\left(x_{3}\right)$, the enzyme substrate complexes $A E_{1}\left(x_{4}\right)$ and $A_{P} E_{2}\left(x_{6}\right)$ and the mono-phosphorylated $A_{P}\left(x_{5}\right)$. The network is composed of 6 reactions, two describing the reversible binding of protein and kinase $E_{1}+A \rightleftharpoons A E_{1}$, one the irreversible release of mono-phosphorylated protein and kinase $A E_{1} \rightarrow E_{1}+A_{P}$, two the reversible binding of mono-phosphorylated protein and phosphatase $E_{2}+A_{P} \rightleftharpoons A_{P} E_{2}$ and one the irreversible release unphosphorylated protein and phosphatase $A_{P} E_{2} \rightarrow E_{2}+A$.

To every reaction we associate a reaction vector describing the stoichiometry of that reaction. For example in the reaction

$$
E_{1}+A \stackrel{k_{1}}{\longrightarrow} A E_{1}
$$

one unit of $A\left(x_{2}\right)$ and $E_{1}\left(x_{1}\right)$ are consumed and one unit of $A E_{1}\left(x_{4}\right)$ is produced, which yields the vector

$$
(-1,-1,0,1,0,0)^{T} .
$$

All in all one obtains six reaction vectors, that we collect as column vectors of the stoichiometric matrix

$$
S^{(1)}=\left[\begin{array}{rrrrrr}
-1 & 1 & 1 & 0 & 0 & 0 \\
-1 & 1 & 0 & 0 & 0 & 1 \\
0 & 0 & 0 & -1 & 1 & 1 \\
1 & -1 & -1 & 0 & 0 & 0 \\
0 & 0 & 1 & -1 & 1 & 0 \\
0 & 0 & 0 & 1 & -1 & -1
\end{array}\right],
$$

where the superscript ${ }^{(1)}$ is used to denote the stoichiometric matrix of a 1-site phosphorylation network. Columns 1 and 2 of $S^{(1)}$ correspond to the reversible binding of $A$ and $E_{1}$, column 3 to the irreversible release of $A_{P}$ and $E_{1}$, columns 4 and 5 to the reversible binding of $A_{P}$ and $E_{2}$ and column 6 to the irreversible release of $A$ and $E_{2}$.

Using mass action kinetics the reaction rate $r_{i}$ (i.e. the speed of reaction $i$ ) is proportional to the product of the concentrations of the educts, for example, the rate $r_{1}=k_{1} x_{1} x_{2}$ for the reaction $E_{1}+A \rightarrow A E_{1}$. Similarly one obtains $r_{2}=k_{2} x_{4}$ for $A E_{1} \rightarrow E_{1}+A, r_{3}=k_{3} x_{4}$ for $A E_{1} \rightarrow$ $E_{1}+A_{P}, r_{4}=l_{1} x_{3} x_{5}$ for $E_{2}+A_{P} \rightarrow A_{P} E_{2}, r_{5}=l_{2} x_{6}$ for $A_{P} E_{2} \rightarrow E_{2}+A_{P}$ and $r_{6}=l_{3} x_{6}$ for 
$A_{P} E_{2} \rightarrow E_{2}+A$. With $x=\left(x_{1}, \ldots, x_{6}\right)^{T}, \kappa=\left(k_{1}, \ldots, k_{3}, l_{1}, \ldots, l_{3}\right)^{T}$ we collect $r_{1}, \ldots, r_{6}$ in the vector

$$
r^{(1)}(\kappa, x)=\left(k_{1} x_{1} x_{2}, k_{2} x_{4}, k_{3} x_{4}, l_{1} x_{3} x_{5}, l_{2} x_{6}, l_{3} x_{6}\right)^{T}
$$

and obtain the dynamical system

$$
\dot{x}=S^{(1)} r^{(1)}(\kappa, x),
$$

which can be written componentwise as

$$
\begin{aligned}
& \dot{x}_{1}=\left(k_{2}+k_{3}\right) x_{4}-k_{1} x_{1} x_{2}, \\
& \dot{x}_{2}=-k_{1} x_{1} x_{2}+k_{2} x_{4}+l_{3} x_{6}, \\
& \dot{x}_{3}=\left(l_{2}+l_{3}\right) x_{6}-l_{1} x_{3} x_{5}, \\
& \dot{x}_{4}=-\left(k_{2}+k_{3}\right) x_{4}+k_{1} x_{1} x_{2}, \\
& \dot{x}_{5}=k_{3} x_{4}-l_{1} x_{3} x_{5}+l_{2} x_{6}, \\
& \dot{x}_{6}=-\left(l_{2}+l_{3}\right) x_{6}+l_{1} x_{3} x_{5} .
\end{aligned}
$$

Note that the vector $r^{(1)}(\kappa, x)$ is fully characterized by the vector $\kappa$ and the exponents of the monomials. We collect those in the rate exponent matrix $\mathcal{Y}^{(1)}$ corresponding to the educt complexes (i.e., the 'left-hand sides' of the reactions):

$$
\mathcal{Y}^{(1)}=\left[\begin{array}{llllll}
1 & 0 & 0 & 0 & 0 & 0 \\
1 & 0 & 0 & 0 & 0 & 0 \\
0 & 0 & 0 & 1 & 0 & 0 \\
0 & 1 & 1 & 0 & 0 & 0 \\
0 & 0 & 0 & 1 & 0 & 0 \\
0 & 0 & 0 & 0 & 1 & 1
\end{array}\right]
$$

Fig. 2 does not consider protein synthesis and degradation, the total amount of protein $A$ is therefore constant, that is, one has the conservation relation

$$
x_{2}+x_{4}+x_{5}+x_{6}=c_{1},
$$

where $c_{1}$ is a constant denoting the total concentration of $A$. The name conservation relation stems form the fact that the above sum of concentrations is constant along solutions of (3b): from (3c) it is easy to see that $\dot{x}_{2}(t)+\dot{x}_{4}(t)+\dot{x}_{5}(t)+\dot{x}_{6}(t)=0$ and hence $x_{2}(t)+x_{4}(t)+x_{5}(t)+x_{6}(t)=$ const. Likewise one has

$$
x_{1}+x_{4}=c_{2} \quad \text { and } \quad x_{3}+x_{6}=c_{3},
$$

with constants $c_{2}$ and $c_{3}$ denoting the total concentration of $E_{1}$ and $E_{2}$, respectively. Conservation relations are defined by elements of the left kernel of the matrix $S^{(1)}$. One obtains, for example, the following full row-rank matrix

$$
Z^{(1)}=\left[\begin{array}{llllll}
1 & 0 & 0 & 1 & 0 & 0 \\
0 & 0 & 1 & 0 & 0 & 1 \\
0 & 1 & 0 & 1 & 1 & 1
\end{array}\right]
$$

with $Z^{(1)} S^{(1)} \equiv 0$ where the rows form a basis of the left kernel of $S^{(1)}$.

3.2. The mass action network derived from Figure 1. The mass action network derived from Fig. 1] (with $n$ an arbitrary but fixed positive number) has been described in [20, 30]. Here we use a similar mathematical description: the network consists of the following chemical species: the protein (substrate) $A$ together with $n$ phosphoforms $A_{P}, \ldots, A_{n P}$; the kinase $E_{1}$ together with $n$ kinase-substrate complexes $A E_{1}, \ldots, A_{n-1 P} E_{1}$ and the phosphatase $E_{2}$ together with $n$ phosphatase-substrate complexes $A_{P} E_{2}, \ldots, A_{n P} E_{2}$. Hence there is a total of $3 n+3$ species. To 


\begin{tabular}{|c|c|c|}
\hline Phos. $\#$ & Species & Var. \\
\hline & $E_{1}$ & $x_{1}$ \\
0 & $A$ & $x_{2}$ \\
& $E_{2}$ & $x_{3}$ \\
\hline \multirow{3}{*}{1} & $A E_{1}$ & $x_{4}$ \\
& $A_{P}$ & $x_{5}$ \\
& $A_{P} E_{2}$ & $x_{6}$ \\
\hline & $\vdots$ & \\
\hline \multirow{3}{*}{$\mathrm{i}$} & $A_{i-1 P} E_{1}$ & $x_{1+3 i}$ \\
& $A_{i P}$ & $x_{2+3 i}$ \\
& $A_{i P} E_{2}$ & $x_{3+3 i}$ \\
\hline & $\vdots$ & \\
\hline & $A_{n-1 P} E_{1}$ & $x_{1+3 n}$ \\
$\mathrm{n}$ & $A_{n P}$ & $x_{2+3 n}$ \\
& $A_{n P} E_{2}$ & $x_{3+3 n}$ \\
\hline
\end{tabular}

TABLE 1. Assignment of variables to species

each species, a variable $x_{i}$ denoting its concentration is assigned as depicted in Table 1. We collect all variables in a $(3 n+3)$-dimensional vector

$$
x:=\left(x_{1}, \ldots, x_{3 n+3}\right)^{T} .
$$

As it will turn out, the chosen labeling entails a simple block structure for the matrices associated to the dynamical system (11b) of the network in Fig. 1, cf., for example, the block structure 15b) for the generators of the nonnegative cone in the kernel of the stoichiometric matrix.

Assuming a distributive mechanism, a single phosphorylation occurs with each encounter of substrate and kinase [13] and $n$ phosphorylations therefore require $n$ encounters of substrate and kinase of the form

$$
E_{1}+A_{i-1 P} \rightleftharpoons A_{i-1 P} E_{1} \longrightarrow E_{1}+A_{i P}, \quad i=1, \ldots, n,
$$

where $A_{0 P}=A$ and $A_{1 P}=A_{P}$. Similarly, $n$ dephosphorylations following a distributive mechanism require $n$ encounters of substrate and phosphatase of the form:

$$
E_{2}+A_{i P} \rightleftharpoons A_{i P} E_{2} \longrightarrow E_{2}+A_{i-1 P}, \quad i=1, \ldots, n .
$$

Each phosphorylation and each dephosphorylation therefore consists of 3 reactions and consequently the network consists of $6 n$ reactions. To each reaction we associate a rate constant. We use $k_{i}$ for phosphorylation and $l_{i}$ for dephosphorylation reactions and obtain the following reaction network:

$$
\begin{array}{r}
E_{1}+A_{i-1 P} \stackrel{k_{3 i-2}}{\underset{k_{3 i-1}}{\rightleftharpoons}} A_{i-1 P} E_{1} \stackrel{k_{3 i}}{\longrightarrow} E_{1}+A_{i P}, i=1, \ldots, n \\
E_{2}+A_{i P} \stackrel{l_{3 i-2}}{\underset{l_{3 i-1}}{\rightleftharpoons}} A_{i P} E_{2} \stackrel{l_{3 i}}{\longrightarrow} E_{2}+A_{i-1 P}, i=1, \ldots, n .
\end{array}
$$

Using this notation, $k_{3 i-2}\left(l_{3 i-2}\right)$ denotes the association constant, $k_{3 i-1}\left(l_{3 i-1}\right)$ the dissociation constant and $k_{3 i}\left(l_{3 i}\right)$ the catalytic constant of the $i$-th phosphorylation (dephosphorylation) step. We collect all rate constants in a vector $\kappa$ defined in the following way: 
Definition 3.1 (The vector of rate constants $\kappa$ ).

We collect the six rate constants associated to the $i$-th phosphorylation/dephosphorylation in network (4) in a sub-vector

$$
\kappa_{(i)}:=\left(k_{3 i-2}, k_{3 i-1}, k_{3 i}, l_{3 i-2}, l_{3 i-1}, l_{3 i}\right)^{T} .
$$

We then combine the sub-vectors to a $6 n$-dimensional column vector

$$
\kappa:=\operatorname{col}\left(\kappa_{(1)}, \ldots, \kappa_{(n)}\right) .
$$

We conclude this subsection with a brief comment on the notation used to denote (sub-)vectors and matrices throughout this contribution.

Remark 3.2 (Vector and matrix notation).

In the following sections the dimension of vectors is determined by the number of phosphorylation sites $n$ according to the formula $3 n+3$. (The vector $x$, for example, lives in Euclidian $\mathbb{R}^{3 n+3}$, cf. Table 1). We will use the symbol $e_{j}$ to denote elements of the standard basis of Euclidian vector spaces and use the superscript ${ }^{(i)}$ to distinguish basis vectors of vector spaces of different dimension $3 i+3$ :

$$
e_{j}^{(i)} \ldots \text { denotes elements of the standard basis of } \mathbb{R}^{3 i+3} \text {. }
$$

Likewise for matrices the superscript ${ }^{(i)}$ is used to indicate that the number of rows and/or columns depends on an integer $i$ (see, for example, equations (10), (8e), (9d) or (15b) below).

Later on we will split vectors of length $3 n+3$ into consecutive sub-vectors of length three and we will use the subscript ${ }_{(i)}$ to denote the $i$-th sub-vector

$$
x_{(i)}:=\left(x_{3 i+1}, x_{3 i+2}, x_{3 i+3}\right)^{T}
$$

and we will use $x=\operatorname{col}\left(x_{(0)}, \ldots, x_{(n)}\right)$ to denote that $x$ consists of $n+1$ such sub-vectors. Likewise we will split vectors of length $6 n$ into sub-vectors

$$
y_{(i)}:=\left(y_{6 i-5}, \ldots, y_{6 i}\right)^{T}
$$

of length six and use $y=\operatorname{col}\left(y_{(1)}, \ldots, y_{(n)}\right)$ to denote that $y$ consists of $n$ such sub-vectors.

3.3. The dynamical system defined by the mass action network (4). Starting with the stoichiometric matrix $S^{(1)}$ and the rate exponent matrix $\mathcal{Y}^{(1)}$ defined by the mass action network depicted in Fig. $2 \mathrm{~b}$ one can recursively construct matrices $S^{(n)}$ and $\mathcal{Y}^{(n)}$ for the network (4). Using the ordering of species and reactions introduced above in Table 1 one obtains $S^{(n)} \in \mathbb{R}^{(3 n+3) \times 6 n}$, $\mathcal{Y}^{(n)} \in \mathbb{R}^{(3 n+3) \times 6 n}$ and $Z^{(n)}$ by the following steps:

(I) Concerning $S^{(n)}$ :

$$
\begin{aligned}
& S^{(1)}=\left[\begin{array}{c}
n_{11} \\
n_{21}
\end{array}\right], \quad S^{(2)}=\left[\begin{array}{c|c}
S^{(1)} & n_{12} \\
& n_{22} \\
\hline \mathbf{0}_{3 \times 6} & n_{21}
\end{array}\right], \quad \ldots \\
& S^{(j)}=\left[\begin{array}{c|c}
S^{(j-1)} & n_{12} \\
& \begin{array}{c}
\mathbf{0}_{3(j-2) \times 6} \\
n_{22}
\end{array} \\
\hline \mathbf{0}_{3 \times 6(j-1)} & n_{21}
\end{array}\right]
\end{aligned}
$$


for $j=3, \ldots, n$ with the following sub-matrices of dimension $3 \times 6$ :

$$
\begin{aligned}
& n_{11}=\left[\begin{array}{rrrrrr}
-1 & 1 & 1 & 0 & 0 & 0 \\
-1 & 1 & 0 & 0 & 0 & 1 \\
0 & 0 & 0 & -1 & 1 & 1
\end{array}\right], \\
& n_{12}=\left[\begin{array}{rrrrrr}
-1 & 1 & 1 & 0 & 0 & 0 \\
0 & 0 & 0 & 0 & 0 & 0 \\
0 & 0 & 0 & -1 & 1 & 1
\end{array}\right], \\
& n_{21}=\left[\begin{array}{rrrrrr}
1 & -1 & -1 & 0 & 0 & 0 \\
0 & 0 & 1 & -1 & 1 & 0 \\
0 & 0 & 0 & 1 & -1 & -1
\end{array}\right], \\
& n_{22}=\left[\begin{array}{rrrrrr}
0 & 0 & 0 & 0 & 0 & 0 \\
-1 & 1 & 0 & 0 & 0 & 1 \\
0 & 0 & 0 & 0 & 0 & 0
\end{array}\right] .
\end{aligned}
$$

For fixed $n$, the recursive formula $(7 \mathrm{~b})$ evaluates to

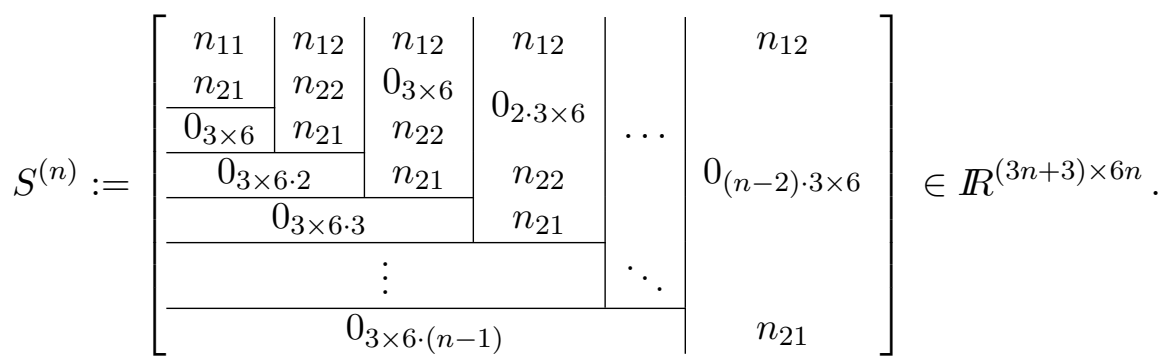

(II) Concerning $\mathcal{Y}^{(n)}$ :

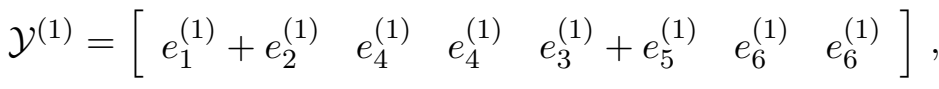

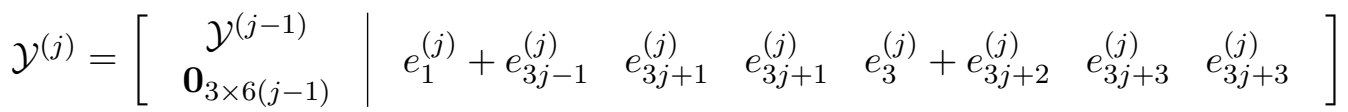

for $j=2, \ldots, n$ with $\mathcal{Y}^{(1)} \in \mathbb{R}^{6 \times 6}$ (cf. eq. $3 \mathrm{~d}$ ) ) and $\mathcal{Y}^{(j)} \in \mathbb{R}^{(3 j+3) \times 6 j}$. Using

$$
Y_{0}(i):=\left[\begin{array}{llllll}
e_{1}^{(i)}+e_{3 i-1}^{(i)} & e_{3 i+1}^{(i)} & e_{3 i+1}^{(i)} & e_{3}^{(i)}+e_{3 i+2}^{(i)} & e_{3 i+3}^{(i)} & e_{3 i+3}^{(i)}
\end{array}\right] \in \mathbb{R}^{(3 i+3) \times 6},
$$

the recursive formula $(9 \mathrm{~b})$ evaluates to

$$
\mathcal{Y}^{(n)^{T}}:=\left[\begin{array}{c|c|c|c|c}
\frac{Y_{0}(1)^{T} \mid 0_{6 \cdot 1 \times 3}}{Y_{0}(2)^{T}} & 0_{6 \cdot 2 \times 3} & 0_{6 \cdot 3 \times 3} & & \\
\hline Y_{0}(3)^{T} & & & \\
\hline & & & \\
\hline Y_{6 \cdot n \times 3}(n-1)^{T} & \ddots & \\
\hline Y_{0}(n)^{T} &
\end{array} .\right.
$$

(III) Concerning $Z^{(n)}$ : 


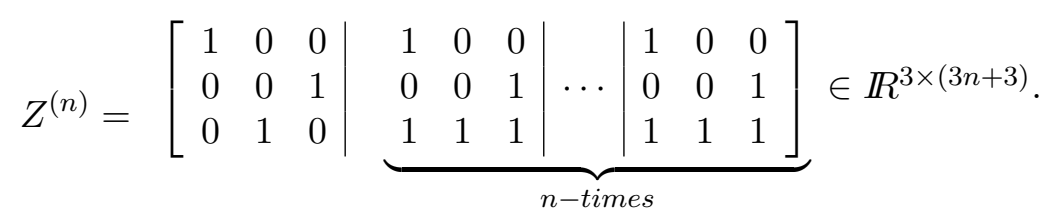

We note that the three rows of $Z^{(n)}$ form a basis for the left kernel of $S^{(n)}$.

Definition 3.3 (Reaction rate vector defined by network (4)).

The vector of rate constants $\kappa$ from $(5 b)$ and the columns $y_{i}$ of $\mathcal{Y}^{(n)}$ from $(9 d)$ define two monomial functions $\Phi^{(n)}: \mathbb{R}^{3 n+3} \rightarrow \mathbb{R}^{6 n}$ and $r^{(n)}(\kappa, x): \mathbb{R}^{3 n+3} \rightarrow \mathbb{R}^{6 n}$ :

$$
\Phi^{(n)}(x):=\left(x^{y_{1}}, \ldots, x^{y_{6 n}}\right)^{T}
$$

and

$$
r^{(n)}(\kappa, x):=\operatorname{diag}(\kappa) \Phi^{(n)}(x)
$$

for $x \in \mathbb{R}_{\geq 0}^{3 n+3}(c f .41)$ ). The $6 n$-dimensional vector $r^{(n)}(\kappa, x)$ is called the reaction rate vector.

Together with the stoichiometric matrix $S^{(n)}$ from $8 \mathrm{e}$, the dynamical system, defined by the network (4) of Fig. 1, is then given by

$$
\dot{x}=S^{(n)} r^{(n)}(\kappa, x)
$$

Remark 3.4 (The monomial function $\Phi^{(n)}(x)$ ).

Observe that the monomial function $\Phi^{(n)}(x)$ in $11 \mathrm{~b}$ satisfies

$$
\begin{aligned}
\Phi^{(n)}\left(e^{\mu}\right) & =e^{\mathcal{Y}^{(n)^{T}} \mu}, \\
\frac{1}{\Phi^{(n)}(x)} & =\Phi^{(n)}\left(x^{-1}\right)
\end{aligned}
$$

for vectors $\mu \in \mathbb{R}^{3 n+3}$ and $x \in \mathbb{R}^{3 n+3}$ with $x_{i} \neq 0, i=1, \ldots, 3 n+3$.

From equation 12 follows that level sets $\left\{x \in \mathbb{R}^{3 n+3} \mid Z^{(n)} x=\right.$ const. $\}$ are invariant under the flow of $(12)$ as $Z^{(n)} x(t)=Z^{(n)} x(0)$ along solutions $x(t)$ of 12 . This observation motivates the classical definition of multistationarity originating in chemical engineering (cf. Figure 3 and, for example, 3 , Definition 1\&Remark 2] or [9, 10]):

Definition 3.5 (Multistationarity).

The system $\dot{x}=S^{(n)} r^{(n)}(\kappa, x)$ from $(12)$ is said to exhibit multistationarity if and only if there exist a positive vector $\kappa \in \mathbb{R}_{>0}^{6 n}$ and at least two distinct positive vectors $a, b \in \mathbb{R}_{>0}^{3 n+3}$ with

$$
\begin{aligned}
S^{(n)} r^{(n)}(\kappa, a) & =0 \\
S^{(n)} r^{(n)}(\kappa, b) & =0 \\
Z^{(n)} a & =Z^{(n)} b .
\end{aligned}
$$

We conclude this section by a discussion of $\operatorname{ker}\left(S^{(n)}\right)$ and of the pointed polyhedral cone $\operatorname{ker}\left(S^{(n)}\right) \cap$ $\mathbb{R}_{\geq 0}^{3 n+3}$ : 


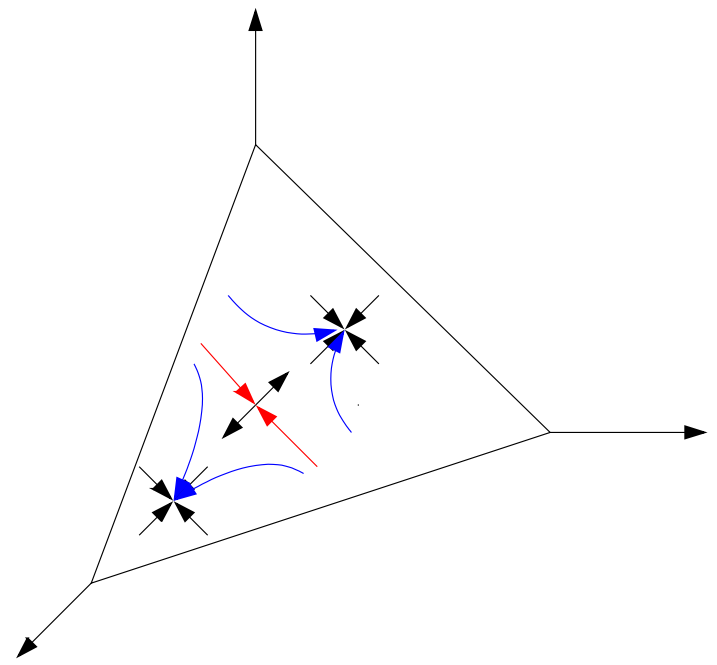

FIGURE 3. Multistationarity and bistability: While the equation $S^{(n)} r^{(n)}(\kappa, x)=0$ often has infinitely many steady state solutions, a solution $x(t)$ of the differential equation $\dot{x}=S^{(n)} r^{(n)}(\kappa, x)$ 'sees' only those contained in the level set $L_{x(0)}:=\{x \in$ $\left.\mathbb{R}_{>0}^{3 n+3} \mid Z^{(n)} x=Z^{(n)} x(0)\right\}$. Multistationarity requires at least two positive steady state solutions in $L_{x(0)}$, bistability two 'stable' positive steady state solutions in $L_{x(0)}$. The triangle in the figure shows such a level set $L_{x(0)}$ in $\mathbb{R}_{\geq 0}^{3}$ with two stable positive steady states. The stable manifold (in red) of the third positive steady state generates the threshold separating the domains of attraction of the two stable steady states.

Lemma 3.6 (A nonnegative basis of $\operatorname{ker}\left(S^{(n)}\right)$ ).

Let $n \geq 2$ be given and recall the stoichiometric matrix $S^{(n)}$ as defined in 8 8e). Let

$$
E=\left[\begin{array}{lll}
1 & 0 & 1 \\
1 & 0 & 0 \\
0 & 0 & 1 \\
0 & 1 & 1 \\
0 & 1 & 0 \\
0 & 0 & 1
\end{array}\right]
$$

and define the $6 n \times 3 n$ matrix with $E$ on the block diagonal (and zero otherwise):

$$
E^{(n)}:=\left[\begin{array}{ccc}
E & & \\
& \ddots & \\
& & E
\end{array}\right]
$$

Then columns of $E^{(n)}$ form a basis of $\operatorname{ker}\left(S^{(n)}\right)$. In addition, the columns of $E^{(n)}$ are generators of $\operatorname{ker}\left(S^{(n)}\right) \cap \mathbb{R}_{\geq 0}^{3 n+3}$.

Proof. First recall the building blocks of $S^{(n)}$, the $3 \times 6$ sub-matrices $n_{11}, n_{21}, n_{12}$ and $n_{22}$ (cf. (8)). We show that $\operatorname{im}\left(E^{(n)}\right)=\operatorname{ker}\left(S^{(n)}\right)$ by proving the two inclusions $\operatorname{im}\left(E^{(n)}\right) \subseteq \operatorname{ker}\left(S^{(n)}\right)$ and $\operatorname{ker}\left(S^{(n)}\right) \subseteq \operatorname{im}\left(E^{(n)}\right)$.

(i) Concerning $\operatorname{im}\left(E^{(n)}\right) \subseteq \operatorname{ker}\left(S^{(n)}\right)$ :

Straightforward computations show that $n_{11} E=0_{3 \times 3}, n_{12} E=0_{3 \times 3}, n_{21} E=0_{3 \times 3}$ and $n_{22} E=$ $0_{3 \times 3}$. Thus $S^{(n)} E^{(n)}=0_{(3 n+3) \times 3 n}$. 
(ii) Concerning $\operatorname{ker}\left(S^{(n)}\right) \subseteq \operatorname{im}\left(E^{(n)}\right)$ :

We first note that the columns of $E$ form a basis of $\operatorname{ker}\left(n_{11}\right)$ and $\operatorname{ker}\left(n_{21}\right)$. Pick any vector $\eta \in \operatorname{ker}\left(S^{(n)}\right)$ and split it in consecutive sub-vectors of length 6 as described in Remark 3.2 $\eta=\operatorname{col}\left(\eta_{(1)}, \ldots, \eta_{(n)}\right)$. The vector $\eta$ satisfies $S^{(n)} \eta=0$, that is (cf. eq. $8 \mathrm{e}$ ) defining $S^{(n)}$ )

$$
\begin{aligned}
n_{11} \eta_{(1)}+\sum_{i=2}^{n} n_{12} \eta_{(i)} & =0, \\
n_{21} \eta_{(1)}+n_{22} \eta_{(2)} & =0, \\
\vdots & \\
n_{21} \eta_{(n-1)}+n_{22} \eta_{(n)} & =0, \\
n_{21} \eta_{(n)} & =0 .
\end{aligned}
$$

From the last equation follows that $\eta_{(n)} \in \operatorname{ker}\left(n_{21}\right)=\operatorname{im}(E)$. The preceding equations then imply $\eta_{(i)} \in \operatorname{ker}\left(n_{21}\right)=\operatorname{im}(E)$, for $i=n-1, \ldots, 1$. Hence $\eta \in \operatorname{im}\left(E^{(n)}\right)$ and thus $\operatorname{ker}\left(S^{(n)}\right) \subseteq \operatorname{im}\left(E^{(n)}\right)$. Finally, concerning the pointed polyhedral cone, observe that a vector $\eta$ is called a generator of the cone $\operatorname{ker}\left(S^{(n)}\right) \cap \mathbb{R}_{\geq 0}^{3 n+3}$, if and only if $\eta$ satisfies the following three conditions (cf. for example, [17]):

$$
\text { (i) } \quad \eta \geq 0, \quad(\text { ii }) \quad S^{(n)} \eta=0
$$

(iii) for generators $\eta_{1}$ and $\eta_{2}$ of $\operatorname{ker}\left(S^{(n)}\right) \cap \mathbb{R}_{\geq 0}^{3 n+3}$ one has $\operatorname{supp}\left(\eta_{1}\right) \subseteq \operatorname{supp}\left(\eta_{2}\right) \Rightarrow \eta_{1}=0$ or $\eta_{1}=\alpha \eta_{2}$ with $\alpha>0$,

where the symbol $\operatorname{supp}(\eta)$ denotes the support of a vector $\eta \in \mathbb{R}^{3 n+3}$ (i.e. the set of indices of the nonzero elements). Observe that the column vectors of $E$ satisfy these conditions and recall the block diagonal structure of $E^{(n)}$. Hence the columns of $E^{(n)}$ satisfy these conditions as well.

\section{RESUlts}

In this section we first introduce a condition for multistationarity (cf. Definition 3.5) in terms of sign patterns, followed by a discussion of sign patterns satisfying this condition. The rather technical proofs of Theorem 4.2 and Theorem 4.10 will be presented in Section 5 and Section 6 respectively.

4.1. A sign condition for multistationarity in the dynamical system (12). We now turn to multistationarity for dynamical systems defined by network (4), that is to the system defined in (12). It follows from Definition 3.5 that multistationarity requires, for a given vector $\kappa$, the existence of two positive solutions $a$ and $b$ to the polynomial equations $114 \mathrm{a}$ ) and (14b) that satisfy the linear condition (14c). In Theorem 4.2 below, we first turn to the polynomial equations. The linear constraint is taken into account afterwards in Corollary 4.5. In the following Theorem 4.2 we use matrices $\Pi^{(n)}$ and $M^{(n)}$ to argue that, for system $\sqrt{12}$, the existence of positive solutions to the polynomial equations is equivalent to the existence of solutions of a linear system.

Definition 4.1 (Matrices $\Pi^{(n)}$ and $\left.M^{(n)}\right)$. Let 1 denote a vector of dimension 6 filled with the number 1 . Define the $6 \times 2$ matrix

$$
\Pi_{0}(i):=[(2-i) \underline{1}(i-1) \underline{1}], i=1, \ldots, n
$$


and the $6 n \times 2$ matrix

$$
\Pi^{(n)}:=\left[\begin{array}{c}
\Pi_{0}(1) \\
\vdots \\
\Pi_{0}(n)
\end{array}\right] .
$$

Further define the $3 \times 3$ matrices

$$
M(0, n):=\left[\begin{array}{ccc}
-1 & -n+1 & n \\
1 & n & -n \\
-1 & -n+2 & n-1
\end{array}\right]
$$

and

$$
M(i, n):=\left[\begin{array}{rrr}
0 & -i+2 & i-1 \\
1 & n-i & -n+i \\
0 & -i+2 & i-1
\end{array}\right]
$$

for $i=1, \ldots, n$ and the $(3 n+3) \times 3$ matrix

$$
M^{(n)}:=\left[\begin{array}{c}
M(0, n) \\
M(1, n) \\
\vdots \\
M(n, n)
\end{array}\right] .
$$

In Section 5 below we prove the following result.

Theorem 4.2 (Solutions to the polynomial equations).

Consider the $n$-site sequential distributed phosphorylation network (4) and recall the corresponding matrices $S^{(n)}, \mathcal{Y}^{(n)}, E^{(n)}, \Pi^{(n)}$ and $M^{(n)}$ (cf. eqns. (8e), (9d), (15b), (16b) and (17c)). The following are equivalent:

(A) There exists a vector $\kappa \in \mathbb{R}_{>0}^{6 n}$ and vectors $a, b \in \mathbb{R}_{>0}^{3 n+3}$ with $a \neq b$ satisfying (14a) \& (14b), that is

$$
S^{(n)} r^{(n)}(\kappa, a)=0, S^{(n)} r^{(n)}(\kappa, b)=0 .
$$

(B) There exist vectors $\mu \in \mathbb{R}^{3 n+3}, \mu \neq 0$, and $(\nu, \lambda) \in \mathbb{R}_{>0}^{3 n} \times \mathbb{R}_{>0}^{3 n}$, such that

$$
\mathcal{Y}^{(n)^{T}} \mu=\ln \frac{E^{(n)} \nu}{E^{(n)} \lambda} .
$$

(C) There exist vectors $\mu \in \mathbb{R}^{3 n+3}, \xi \in \mathbb{R}^{2}$ and $\nu, \lambda \in \mathbb{R}_{>0}^{6 n} \times \mathbb{R}_{>0}^{6 n}$, with $\mu \neq 0$ such that

(a) the vectors $\mu$ and $\xi$ satisfy

$$
\mathcal{Y}^{(n)^{T}} \mu=\Pi^{(n)} \xi,
$$

(b) and the vectors $\nu, \lambda$ and $\xi$ satisfy

$$
\lambda \in \mathbb{R}_{>0}^{6 n}, \quad \text { free },
$$

and, for $i=1, \ldots, n$

$$
\begin{aligned}
\nu_{3 i} & =\lambda_{3 i} e^{(2-i) \xi_{1}+(i-1) \xi_{2}}, \\
\nu_{3 i-2} & =\lambda_{3 i-2} \frac{\nu_{3 i}}{\lambda_{3 i}}, \\
\nu_{3 i-1} & =\lambda_{3 i-1} \frac{\nu_{3 i}}{\lambda_{3 i}} .
\end{aligned}
$$


(D) There exists a vector $\mu \in \mathbb{R}^{3 n+3}, \mu \neq 0$ with

$$
\mu \in \operatorname{im}\left(M^{(n)}\right) .
$$

Remark 4.3 (Solutions $a, b$ and $\kappa$ to the polynomial equations $14 a$ (14) $\&(14 b)$ ).

Recall the matrices $M^{(n)}$ and $E^{(n)}$ (cf. eq. $\sqrt{17 \mathrm{c}}$ ) and eq. $\left.15 \mathrm{~b}\right)$ ) and the monomial function $\Phi^{(n)}$ from eq. (11b) with the properties (13a) and (13b) stated in Remark 3.4. Fix any two vectors $\mu \in \operatorname{im}\left(M^{(n)}\right)$ and $\lambda \in \mathbb{R}_{>0}^{6 n}$. Then vectors $(\kappa, a)$ and $(\kappa, b)$ satisfying the polynomial equations (14a) \& (14b) are determined by

$$
\operatorname{diag}(\kappa) \Phi^{(n)}\left(e^{\ln a}\right)=E^{(n)} \nu, \quad \operatorname{diag}(\kappa) \Phi^{(n)}\left(e^{\ln b}\right)=E^{(n)} \lambda, \quad(\nu, \lambda) \in \mathbb{R}_{>0}^{3 n} \times \mathbb{R}_{>0}^{3 n} .
$$

By (13a), they fulfill 18 for $\mu=\ln \left(\frac{a}{b}\right)$. Thus, vectors $(\kappa, a)$ and $(\kappa, b)$ given by

$$
\begin{aligned}
& a, \text { free in } \mathbb{R}_{>0}^{3 n+3}, \\
& b:=\operatorname{diag}\left(e^{\mu}\right) a, \\
& \kappa:=\operatorname{diag}\left(\Phi^{(n)}\left(a^{-1}\right)\right) E^{(n)} \lambda .
\end{aligned}
$$

are solutions to the polynomial equations $14 \mathrm{a}) \&(14 \mathrm{~b}$ ). Note that, in particular, the vector $a \in$ $\mathbb{R}_{>0}^{3 n+3}$ is free and that $a$ and $b$ satisfy by construction

$$
\ln b-\ln a=\mu \in \operatorname{im}\left(M^{(n)}\right) .
$$

This follows from the proof of Theorem 4.2, given in Section 5 below. See also [3, Remark 7]. In Section 7. we will interpret the fact that eq. (17b) implies $\mu_{3 i+1}=\mu_{3 i+3}$ in eq. (22) for $i=1,2,, \ldots, n$.

As a consequence of Theorem 4.2, any two distinct solutions $a$ and $b$ to the polynomial equations (14a) \& (14b) satisfy the condition (22) with $\mu \neq 0$. And vice versa in case $\kappa$ is chosen according to (21c). From Definition 3.5 follows that for multistationarity $a$ and $b$ additionally have to satisfy $Z^{(n)}(b-a)=0$ and hence $b-a \in \operatorname{im}\left(S^{(n)}\right)$. The existence of vectors $a$ and $b$ satisfying both conditions is covered by Corollary 4.5 given below (this result itself is a consequence of [4, Lemma 1]; see also [3] and, for example, [10] for an earlier, more informal discussion). The corollary is based on the sign patterns of vectors and sets thereof.

Definition 4.4 (Sign patterns defined by vectors and linear subspaces). Given $v \in \mathbb{R}^{m}$, a vector $\sigma \in\{-1,0,1\}^{m}$ with

$$
\sigma_{i}=\operatorname{sign}\left(v_{i}\right)
$$

is called the sign pattern of $v: \sigma=\operatorname{sign}(v)$. Given a linear subspace $V \subseteq \mathbb{R}^{m}$, the set $\operatorname{sign}(V)$ of sign patterns is defined by

$$
\operatorname{sign}(V):=\left\{\sigma \in\{-1,0,1\}^{m} \mid \exists v \in V \quad \text { with } \quad \sigma=\operatorname{sign}(v)\right\} .
$$

Now we can state the announced result:

Corollary 4.5 (cf. Lemma 1 of [4]).

Two vectors $a, b \in \mathbb{R}_{>0}^{3 n+3}$ with

$$
\begin{aligned}
a & \neq b \\
\ln b-\ln a & \in \operatorname{im}\left(M^{(n)}\right) \\
b-a & \in \operatorname{im}\left(S^{(n)}\right)
\end{aligned}
$$


exist if and only if

$$
\mathcal{M}^{(n)}:=\operatorname{sign}\left(\operatorname{im}\left(M^{(n)}\right)\right) \cap \operatorname{sign}\left(\operatorname{im}\left(S^{(n)}\right)\right) \neq\{0\} .
$$

In case (25) holds, every pair $\mu \in \operatorname{im}\left(M^{(n)}\right), s \in \operatorname{im}\left(S^{(n)}\right)$ with

$$
\operatorname{sign}(\mu)=\operatorname{sign}(s) \neq 0
$$

defines a pair of positive vectors $a$ and $b$ with the property (24) via:

$$
a=\left(a_{i}\right)_{i=1, \ldots, 3 n+3}
$$

with

$$
\begin{aligned}
a_{i} & =\left\{\begin{array}{l}
\frac{s_{i}}{e^{\mu_{i}}-1}, \text { if } \mu_{i} \neq 0 \\
\bar{a}_{i}>0, \text { free },
\end{array}\right. \\
b & =\operatorname{diag}\left(e^{\mu}\right) a .
\end{aligned}
$$

Proof. Apply [4, Lemma 1] with $a$ as $p, b$ as $q$, im $\left(M^{(n)}\right)$ as $M_{1}$ and $\operatorname{im}\left(S^{(n)}\right)$ as $M_{2}$.

As a consequence one can state a condition for multistationarity in system $(12$ defined by the network 44 based on the sign patterns defined by the linear subspaces $\operatorname{im}\left(S^{(n)}\right)$ and $\operatorname{im}\left(M^{(n)}\right)$ and the set $\mathcal{M}^{(n)}$. We will argue in Remark 4.7 that this condition is satisfied if and only if at least one out of $\frac{1}{2}\left(3^{3 n+3}-1\right)$ linear systems is feasible. Hence one may in fact establish multistationarity for the polynomial system 12 by an analysis of linear inequality systems.

Theorem 4.6 (A sign condition for multistationarity of systems (12)).

Consider the $n$-site sequential distributed phosphorylation network (4) and recall the corresponding matrices $S^{(n)}, Z^{(n)}$ and $M^{(n)}$, (cf. eqns. (8e), (10)) and $(17 c)$. There exists vectors $a, b \in \mathbb{R}_{>0}^{3 n+3}$ and $\kappa \in \mathbb{R}_{>0}^{6 n}$ satisfying

$$
S^{(n)} r^{(n)}(\kappa, a)=0, S^{(n)} r^{(n)}(\kappa, b)=0, Z^{(n)}(b-a)=0,
$$

if and only if

$$
\mathcal{M}^{(n)}=\operatorname{sign}\left(\operatorname{im}\left(M^{(n)}\right)\right) \cap \operatorname{sign}\left(\operatorname{im}\left(S^{(n)}\right)\right) \neq\{0\} .
$$

Proof. Suppose that $\mathcal{M}^{(n)} \neq\{0\}$. Then, by Corollary 4.5 vectors $a$ and $b$ satisfying $24 a-(24 c)$ are given by $27 \mathrm{a})-(27 \mathrm{c})$. By Remark 4.3 (where $a>0$ is free and may be chosen as in (27a) \& (27b)) and Theorem 4.2 there exists a vector $\kappa \in \mathbb{R}_{>0}^{6 n}$ given by eq. 21c such that $a, b$ and $\kappa$ are solutions of the polynomials $(14 \mathrm{a}) \&(14 \mathrm{~b})$. By $24 \mathrm{c}) a$ and $b$ additionally satisfy $Z^{(n)}(b-a)=0$. Vice versa, let $a, b \in \mathbb{R}_{>0}^{3 n+3}$ with $a \neq b$ and $\kappa \in \mathbb{R}_{>0}^{6 n}$ be given, such that 1 (14a), (14b) \& $14 \mathrm{c}$ hold. From eq. 20 of Theorem 4.2 follows that $\mu:=\ln b-\ln a \in \operatorname{im}\left(M^{(n)}\right)$ (cf. also Remark 4.3 . eq. (22) ) and, as $Z^{(n)}(b-a)=0 \Leftrightarrow b-a \in \operatorname{im}\left(S^{(n)}\right)$, Corollary 4.5 implies that 25 holds.

Remark 4.7 (The condition $\mathcal{M}^{(n)} \neq\{0\}$ ).

(A) $\mathcal{M}^{(n)} \neq\{0\}$ is equivalent to feasibility of (at least) one linear system: We emphasize the relation between condition $(25)$ and linear inequality systems. An element $\sigma \in\{-1,0,1\}^{3 n+3}$ is an element of $\mathcal{M}^{(n)}$ from (25) if and only if the following linear system is feasible:

$\exists s \in \mathbb{R}^{3 n+3}$ and $\xi \in \mathbb{R}^{3}$ such that

$$
\begin{aligned}
& Z^{(n)} s=0, \text { with } \sigma_{i} s_{i}>0 \text { if } \sigma_{i} \neq 0 \text { and } s_{i}=0 \text { else, and } \\
& \mu=M^{(n)} \xi, \text { with } \sigma_{i} \mu_{i}>0 \text { if } \sigma_{i} \neq 0 \text { and } \mu_{i}=0 \text { else. }
\end{aligned}
$$


(B) Elements of $\{-1,1\}^{3 n+3}$ : Recall the formulae $27 \mathrm{a}-27 \mathrm{c}$ and observe that $\mu_{i}=0 \Leftrightarrow a_{i}=b_{i}$. Hence a vector $\mu$ (and corresponding $s$ ) with $\operatorname{sign}(\mu) \in\{-1,1\}^{3 n+3}$ yields $a$ and $b$ that differ in every coordinate, while a vector $\mu$ (and corresponding $s$ ) with sign $(\mu) \in\{-1,0,1\}^{3 n+3}, \mu \neq 0$ yields $a$ and $b$ that may be equal in some coordinates.

(C) Testing $\mathcal{M}^{(n)} \neq\{0\}:$ Note that there are $3^{3(n+1)}\left(2^{3(n+1)}\right)$ sign patterns $\sigma \in\{-1,0,1\}^{3 n+3}$ $\left(\sigma \in\{-1,1\}^{3 n+3}\right)$. A naive algorithm for computing nontrivial elements $\sigma$ of $\mathcal{M}^{(n)}$ from (25) would therefore be to enumerate $\frac{1}{2}\left(3^{(3 n+3)}-1\right)\left(2^{(3 n+2)}\right)$ of these sign patterns and check feasibility of every linear system (28) associated to one of those. (As feasibility of (28) for an element $\sigma$ implies feasibility for $-\sigma$, one has to check only half of the sign patterns.) For the general problem of computing all sign patterns defined by two linear subspaces, a more advanced algorithm involving mixed integer linear programming is described in the thesis [29]. This software has aided the discovery of the sign patterns described below.

Before turning to the elements of $\mathcal{M}^{(n)}$, we conclude this section by examining the linear system (28) for $n=2$ :

Example 4.8 (Steady states and corresponding rate constants for $n=2$ via the system (28)). From $17 \mathrm{a})$ - $17 \mathrm{c}$ and 10 we obtain

$$
M^{(2)}=\left[\begin{array}{rrr}
-1 & -1 & 2 \\
1 & 2 & -2 \\
-1 & 0 & 1 \\
\hline 0 & 1 & 0 \\
1 & 1 & -1 \\
0 & 1 & 0 \\
\hline 0 & 0 & 1
\end{array}\right] \text { and } Z^{(2)}=\left[\begin{array}{lll|lll|lll}
1 & 0 & 0 & 1 & 0 & 0 & 1 & 0 & 0 \\
0 & 0 & 1 & 0 & 0 & 1 & 0 & 0 & 1 \\
0 & 1 & 0 & 1 & 1 & 1 & 1 & 1 & 1
\end{array}\right] \text {. }
$$

We observe that, for the sign pattern

$$
\sigma^{(2)}:=(1, \quad 1, \quad 1|1,-1, \quad 1|-1,-1,-1)^{T},
$$

the columns of the matrices

$$
B=\left[\begin{array}{rrr}
1 & 0 & 0 \\
0 & 1 & 0 \\
2 & 1 & 1 \\
\hline 1 & 1 & 0 \\
-1 & 0 & -1 \\
1 & 1 & 0 \\
\hline 0 & 0 & -1 \\
-2 & -1 & -2 \\
0 & 0 & -1
\end{array}\right] \text { and } C=\left[\begin{array}{rrr|rrr}
1 & 0 & 0 & 0 & 0 & 0 \\
1 & 1 & 0 & 1 & 0 & 1 \\
0 & 1 & 0 & 0 & 0 & 0 \\
\hline 0 & 0 & 1 & 0 & 0 & 0 \\
0 & 0 & 0 & -1 & 0 & 0 \\
0 & 0 & 0 & 0 & 1 & 0 \\
\hline-1 & 0 & -1 & 0 & 0 & 0 \\
0 & 0 & 0 & 0 & 0 & -1 \\
0 & -1 & 0 & 0 & -1 & 0
\end{array}\right]
$$

form a basis of $\operatorname{im}\left(M^{(2)}\right)$ and of $\operatorname{ker}\left(Z^{(2)}\right)$ respectively. The expressions

$$
\mu=B \beta \in \operatorname{im}\left(M^{(2)}\right), \beta \in \mathbb{R}_{>0}^{3}, \text { and } s=C \gamma \in \operatorname{ker}\left(Z^{(2)}\right), \gamma \in \mathbb{R}_{>0}^{6},
$$

define feasible vectors for 28 with the given sign pattern $\sigma^{(2)}$. Hence $\sigma^{(2)}$ defines a feasible system 28): $\sigma^{(2)} \in \mathcal{M}^{(2)}$. For a numerical example, we choose

$$
\begin{gathered}
\beta_{1}=\ln (3 / 2), \beta_{2}=\ln (4 / 3), \beta_{3}=\frac{\ln 2}{2}, \\
\gamma_{1}=1, \gamma_{2}=1 / 2, \gamma_{3}=1, \gamma_{4}=1 / 4, \gamma_{5}=1, \gamma_{6}=1 / 4 .
\end{gathered}
$$

Using these $\beta \in \mathbb{R}_{>0}^{3}$ and $\gamma \in \mathbb{R}_{>0}^{6}$, one obtains the vectors $\mu$ and $s$ given in Table 2. Hence, by (27a), 27b) and (27c), one arrives at the steady states $a$ and $b$ as given in Table 2. The associated 


\begin{tabular}{|c|c|c|c|}
\hline$\mu$ & $s$ & $a$ & $b$ \\
\hline $\ln \frac{3}{2}$ & 1 & 2 & 3 \\
$\ln \frac{4}{3}$ & 2 & 6 & 8 \\
$-\frac{\ln 2}{2}+\ln 6$ & $\frac{1}{2}$ & $\frac{1}{34}(1+3 \sqrt{2})$ & $\frac{3}{34}(6+\sqrt{2})$ \\
$\ln 2$ & 1 & 1 & 2 \\
$\ln \frac{\sqrt{2}}{3}$ & $-\frac{1}{4}$ & $\frac{3}{28}(3+\sqrt{2})$ & $\frac{1}{28}(2+3 \sqrt{2})$ \\
$\ln 2$ & 1 & 1 & 2 \\
$-\frac{\ln 2}{2}$ & -2 & $2(2+\sqrt{2})$ & $2(1+\sqrt{2})$ \\
$-\ln 6$ & $-\frac{1}{4}$ & $\frac{3}{10}$ & $\frac{1}{20}$ \\
$-\frac{\ln 2}{2}$ & $-\frac{3}{2}$ & $3+\frac{3}{\sqrt{2}}$ & $\frac{3}{2}(1+\sqrt{2})$ \\
\hline
\end{tabular}

TABLE 2. Columns 1\&2: vectors $\mu$ and $s$ with $\operatorname{sign}(\mu)=\operatorname{sign}(s)$ satisfying the system (28) (with $n=2$ and $\sigma^{(2)}$ ). Columns 3\&4: Steady state vectors $a$ and $b$ computed from $\mu$ and $s$ via (27a), 27b) \& (27c). Simple computation shows $c=Z^{(2)} a=Z^{(2)} b=\left(7+2 \sqrt{2}, \frac{1}{34}(137+54 \sqrt{2}), \frac{1}{140}(2187+505 \sqrt{2})\right)^{T}$.

\begin{tabular}{|c|c|c|c|c|c|c|c|c|c|c|c|}
\hline$k_{1}$ & $k_{2}$ & $k_{3}$ & $l_{1}$ & $l_{2}$ & $l_{3}$ & $k_{4}$ & $k_{5}$ & $k_{6}$ & $l_{4}$ & $l_{5}$ & $l_{6}$ \\
\hline $5 \sqrt{2}$ & $30 \sqrt{2}$ & $30 \sqrt{2}$ & 952 & 27 & $30 \sqrt{2}$ & $\frac{4}{3}(4+\sqrt{2})$ & $\frac{1}{2}$ & $\frac{1}{2}$ & $\frac{40}{3}(4+5 \sqrt{2})$ & $\frac{2}{3}$ & $\frac{2}{3}$ \\
\hline
\end{tabular}

TABLE 3. One choice of rate constants such that $a$ and $b$ from Table 2 are steady states (i.e. $\kappa=\left(k_{1}, k_{2}, k_{3}, l_{1}, l_{2}, l_{3}, k_{4}, k_{5}, k_{6}, l_{4}, l_{5}, l_{6}\right)^{T}$ with $S^{(2)} r^{(2)}(\kappa, a)=$ $\left.S^{(2)} r^{(2)}(\kappa, b)=0\right)$.

rate constant vectors $\kappa$ are obtained by $21 \mathrm{c})$ for the value of $a$ from Table 2 and for free $\lambda \in \mathbb{R}_{>0}^{6}$. Here we choose

$$
\lambda=(30 \sqrt{2}, 27,30 \sqrt{2}, 2+\sqrt{2}, 2+\sqrt{2}, 2+\sqrt{2})^{T}
$$

to obtain the rate constants given in Table 3. For the illustration in Fig. 4, a numerical continuation of the steady state $a$ has been performed using the rate constants from Table 3. The numerical continuation reveals that, for the rate constants of Table 3 and the total concentrations $c=Z^{(2)} a=$ $Z^{(2)} b$ defined by either $a$ or $b$, the system has three steady states, namely $a, b$ and

$$
\tilde{b}=(1.52 . ., 1.01 . ., 0.01 . ., 0.12 . ., 0.74 . ., 0.12 . ., 8.17 . ., 4.39 . ., 6.13 . .)^{T} \text {. }
$$

A local stability analysis shows the exponential stability of $b$ and $\tilde{b}$. The steady state $a$ turns out to be a saddle point because of the presence of exactly one simple positive eigenvalue. Hence the system shows bistability as indicated in Figure 3. By the results of [30], these are all steady states of the double phosphorylation system once $\beta, \gamma$ and $\lambda$ are fixed.

4.2. Sign patterns for feasibility of system $(\mathbf{2 8})$. Given a $\sigma^{(n)} \in\{-1,1\}^{3 n+3}$, we first turn to the subproblem $Z^{(n)} s=0$ with sign $(s)=\sigma^{(n)}$ in $(28)$. To this end, we consider sign patterns in column and matrix form. With $(n+1)$ column vectors $\tau_{(0)}=\left(\tau_{10}, \tau_{20}, \tau_{30}\right)^{T}, \ldots, \tau_{(n)}=\left(\tau_{1 n}, \tau_{2 n}, \tau_{3 n}\right)^{T}$ from $\{-1,1\}^{3}$ we associate the sign pattern matrix

$$
\tau=\left(\tau_{(0)} \cdots \tau_{(n)}\right) \in\{-1,1\}^{3 \times(n+1)} .
$$

and the sign pattern column $\tau^{(n)}=\operatorname{col}(\tau)=\operatorname{col}\left(\tau_{(0)}, \ldots, \tau_{(n)}\right) \in\{-1,1\}^{3 n+3}$. 


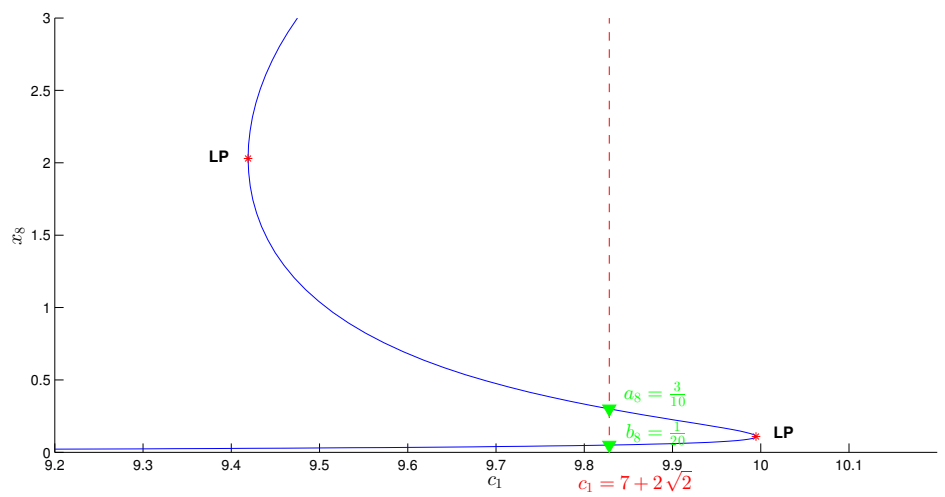

Figure 4. Numerical continuation starting in $a$ from Table 3 . Here $x_{8}$ (i.e. the concentration of $A_{2 P}$ ) is plotted against $c_{1}$ (i.e. the total concentration of kinase $\left.E_{1}\right)$. Green triangles mark the components $a_{8}$ and $b_{8}$ of the steady state vectors $a$ and $b$ from Table 2 , the total concentration $c_{1}$ at $a$ (and $b$ ) is displayed in red. The component $\tilde{b}_{8}$ of the third steady state is close to 4.39 .

Lemma 4.9 ( Feasibility of $Z^{(n)} s=0$ and $\operatorname{sign}(s)=\sigma^{(n)} \in\{-1,1\}^{3 n+3}$ ). For a given sign pattern matrix

$$
\sigma=\left(\sigma_{(0)}, \ldots, \sigma_{(n)}\right)=\left(\begin{array}{ccc}
\sigma_{10} & \ldots & \sigma_{1 n} \\
\sigma_{20} & \ldots & \sigma_{2 n} \\
\sigma_{30} & \ldots & \sigma_{3 n}
\end{array}\right) \in\{-1,1\}^{3 \times(n+1)}
$$

and its associated sign pattern vector $\sigma^{(n)}=\operatorname{col}\left(\sigma_{(0)}, \ldots, \sigma_{(n)}\right) \in\{-1,1\}^{3 n+3}$, the subproblem

$$
Z^{(n)} s=0 \text { with } \operatorname{sign}(s)=\sigma^{(n)}
$$

in (28) is not feasible if and only if $\sigma$ has one of the following three properties:

(1) The elements of the first row are all of the same sign $\sigma_{10}$.

(2) The elements of the second row are all of the same sign $\sigma_{20}$ with $\sigma_{20} \sigma_{10}<0$ and $\sigma_{20} \sigma_{30}<0$.

(3) The elements of the third row are all of the same sign $\sigma_{30}$.

Proof. In analogy to $\sigma^{(n)}$, we use the notation $s=\left(s_{10}, s_{20}, s_{30}, s_{11}, s_{21}, s_{31}\right.$, $\left., s_{1 n}, s_{2 n}, s_{3 n}\right)^{T}$ The claim follows easily from the fact that $Z^{(n)} s=0$ is equivalent to the decoupled linear system

$$
\sum_{j=0}^{n} s_{1 j}=0, \quad \sum_{j=0}^{n} s_{3 j}=0, \quad \sum_{j=0}^{n} s_{2 j}=s_{10}+s_{30}
$$

(cf. the definition of $Z^{(n)}$ in eq. 10$)$ ).

We now turn to those sign patterns $\sigma^{(n)} \in\{-1,1\}^{3 n+3}$, where 31 is feasible and where, additionally, the second subproblem

$$
\mu=M^{(n)} \xi \text { with } \operatorname{sign}(\mu)=\sigma^{(n)}
$$

is feasible. In particular, we will show that (31) and $(32)$ are only feasible for sign patterns involving

$$
\begin{array}{lll}
\sigma_{1}:=(+1,+1,+1)^{T}, & \sigma_{2}:=(+1,-1,+1)^{T}, & \sigma_{3}:=(-1,-1,-1)^{T}=-\sigma_{1} \\
\sigma_{4}:=(+1,-1,-1)^{T}, & \sigma_{5}:=(-1,+1,-1)^{T}=-\sigma_{2}, & \sigma_{6}:=(-1,+1,+1)^{T}=-\sigma_{4},
\end{array}
$$


while it is infeasible for sign patterns involving

$$
\sigma_{7}:=(+1,+1,-1)^{T}, \sigma_{8}:=(-1,-1,+1)^{T}=-\sigma_{7} .
$$

We use these $\sigma_{j}$ to encode sign patterns $\sigma^{(n)} \in\{-1,1\}^{3 n+3}$. For example, for $n=2$ we encode

$$
\sigma^{(2)}:=(1, \quad 1, \quad 1|1,-1, \quad 1|-1,-1,-1)^{T} \in \mathbb{R}^{9}
$$

as the $3 \times 3$ array

$$
\sigma=\left(\sigma_{1} \sigma_{2} \sigma_{3}\right)
$$

with the columns $\sigma_{1}, \sigma_{2}$ and $\sigma_{3}$. We use the symbol $[\tau]_{i}$ as shorthand notation for the $i$-fold concatenation of $\tau \in\{-1,1\}^{3}$, i.e., for the $3 \times i$-matrix

$$
[\tau]_{i}=(\underbrace{\tau \ldots \tau}_{i \text { times }})
$$

For an example with $n=5$,

$$
\sigma=\left(\left[\sigma_{3}\right]_{3}\left[\sigma_{5}\right]_{2} \sigma_{1}\right)=\left(\sigma_{3} \sigma_{3} \sigma_{3} \sigma_{5} \sigma_{5} \sigma_{1}\right)
$$

stands for the 18-dimensional sign pattern

$$
\sigma^{(5)}=(-1,-1,-1|-1,-1,-1|-1,-1,-1|-1,+1,-1|-1,+1,-1 \mid+1,+1,+1)^{T} .
$$

In the following Theorem 4.10 we characterize the sign patterns $\sigma^{(n)} \in\{-1,1\}^{3 n+3}$ that are feasible for (28) for $n \geq 2$. In Section 6 below we will prove this result.

Theorem 4.10. Recall the linear system (28) associated to the sign pattern vector $\sigma^{(n)} \in\{-1,1\}^{3 n+3}$ and hence to the equivalent sign pattern matrix $\sigma \in\{-1,1\}^{3 \times(n+1)}$. For $n \geq 2$ the linear systems 28) associated to $\sigma^{(n)}$ and to $-\sigma^{(n)}$ are feasible if and only if $\sigma$ is one of the following sign patterns (s) to $\left.s_{7}\right)$ :

- For $n \geq 2$ :

$$
\begin{aligned}
\sigma & =\left(\sigma_{2}\left[\sigma_{3}\right]_{i_{1}}\left[\sigma_{1}\right]_{i_{2}}\right) \text { with } i_{1}, i_{2} \geq 1 \text { and } i_{1}+i_{2}=n \\
\sigma & =\left(\sigma_{3}\left[\sigma_{5}\right]_{i_{1}}\left[\sigma_{1}\right]_{i_{2}}\right) \text { with } i_{1}, i_{2} \geq 1 \text { and } i_{1}+i_{2}=n \\
\sigma & =\left(\sigma_{4}\left[\sigma_{5}\right]_{i_{1}}\left[\sigma_{1}\right]_{i_{2}}\right) \text { with } i_{1}=1, i_{2} \geq 1 \text { and } i_{1}+i_{2}=n \\
\sigma & =\left(\sigma_{4}\left[\sigma_{3}\right]_{i_{1}}\left[\sigma_{1}\right]_{i_{2}}\right) \text { with } i_{1}, i_{2} \geq 1 \text { and } i_{1}+i_{2}=n
\end{aligned}
$$

- Additionally for $n \geq 3$ :

$$
\begin{aligned}
& \sigma=\left(\sigma_{2}\left[\sigma_{3}\right]_{i_{1}}\left[\sigma_{2}\right]_{i_{2}}\left[\sigma_{1}\right]_{i_{3}}\right) \text { with } i_{1}, i_{2}, i_{3} \geq 1 \text { and } i_{1}+i_{2}+i_{3}=n \\
& \sigma=\left(\sigma_{3}\left[\sigma_{3}\right]_{i_{1}}\left[\sigma_{5}\right]_{i_{2}}\left[\sigma_{1}\right]_{i_{3}}\right) \text { with } i_{1}, i_{2}, i_{3} \geq 1 \text { and } i_{1}+i_{2}+i_{3}=n \\
& \sigma=\left(\sigma_{4}\left[\sigma_{3}\right]_{i_{1}}\left[\sigma_{5}\right]_{i_{2}}\left[\sigma_{1}\right]_{i_{3}}\right) \text { with } i_{1}, i_{3} \geq 1, i_{2}=1 \text { and } i_{1}+i_{2}+i_{3}=n .
\end{aligned}
$$

By Remark 4.7 (A), $\sigma^{(n)}$ is a nontrivial element of $\mathcal{M}^{(n)}$ from 25 if and only if $\sigma^{(n)}$ is one of the sign patterns $\left(s_{1}\right)$ to $\left(s_{7}\right)$ or its negative. As an immediate consequence of Theorem 4.10 above we note the following:

Remark $4.11\left(M^{(n)} \neq\{0\}\right.$ : multistationarity in $n$-site sequential distributive phosphorylation for $n \geq 2$ ).

As a consequence of Theorem 4.6 and 4.10 we conclude that for every $n \geq 2$ there exists a vector of rate constants $\kappa$ such that the system (14a), (14b) \& 14c) has at least two distinct positive solutions $a$ and $b$. Every element $\sigma$ described in Theorem 4.10 defines a feasible linear system of the form (28). And solutions thereof parameterize the steady states $a$ and $b$ and the vectors $\kappa$ of rate constants via the equations (27a), 27b) and (21c) respectively (cf. Example 4.8). It will be interesting to systematically explore the corresponding regions in the rate constant space, especially with respect to biological plausibility and function. 
Remark $4.12\left(\mathcal{M}^{(1)}=\{0\}\right.$ excludes multistationarity for $n=1$ (single phosphorylation)). Any $\mu \in \operatorname{im}\left(M^{(1)}\right)$ and any $s \in \operatorname{ker}\left(Z^{(1)}\right)$ can be written as

$$
\mu=\left(\mu_{1}, \mu_{4}-\mu_{1}, \mu_{3}, \mu_{4}, \mu_{4}-\mu_{1}, \mu_{4}\right)^{T} \text { and } s=\left(s_{1}, s_{2}, s_{3},-s_{1}, s_{3}-s_{2}+s_{1},-s_{3}\right)^{T}
$$

respectively. We assume $\operatorname{sign}(\mu)=\operatorname{sign}(s)$. In case of $\mu_{4}=0$ we arrive at $\mu=0=s$. In case of $\mu_{4} \neq 0$ we assume without loss of generality $\mu_{4}>0$ and are led to the sign pattern $\sigma^{(1)}=(-,+,-,+,+,+)^{T}$ with sign pattern matrix

$$
\sigma=\left(\begin{array}{ll}
- & + \\
+ & + \\
- & +
\end{array}\right) .
$$

By Lemma 4.9 these sign patterns cannot belong to an element of $\operatorname{ker}\left(Z^{(1)}\right)$ and we conclude $\mathcal{M}^{(1)}=\{0\}$.

Finally we want to count the number of sign patterns of the form $\left(s_{1}\right)-\left(s_{7}\right)$. To demonstrate the reasoning we first examine sign patterns of the form $\left(s_{2}\right)$ for $n=2, \ldots, 5$ :

Example 4.13 (The sign pattern $\left(s_{2}\right)$ ).

For $2 \leq n \leq 5$ one obtains from the formula $\left(s_{2}\right.$ the following patterns:

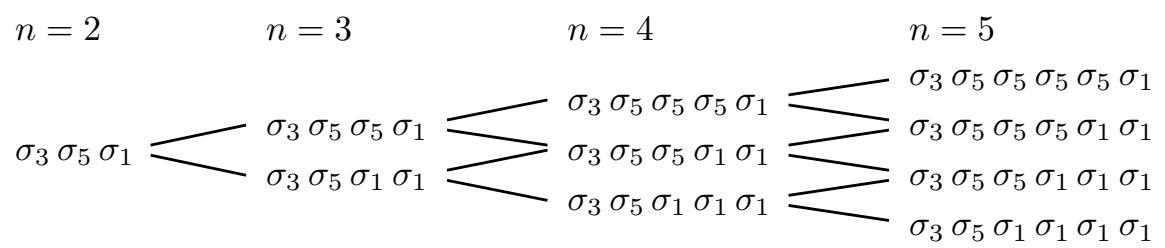

We summarize for $s_{2}$ in case of $n=5$ :

- The integer 5 can be partitioned in the sum of two integers $i_{1}, i_{2}$ in the following two ways: $5=1+4=3+2$. As we have to take the ordering of $i_{1}$ and $i_{2}$ into account (and hence, for example, $(4,1)$ differs from $(1,4))$ we obtain the following pairs of integers:

$$
\{(1,4),(4,1),(3,2),(2,3)\} \text {. }
$$

- This yields the sign patterns

$$
\sigma_{3}\left[\sigma_{5}\right]_{4}\left[\sigma_{1}\right]_{1}, \quad \sigma_{3}\left[\sigma_{5}\right]_{1}\left[\sigma_{1}\right]_{4}, \quad \sigma_{3}\left[\sigma_{5}\right]_{3}\left[\sigma_{1}\right]_{2}, \quad \sigma_{3}\left[\sigma_{5}\right]_{2}\left[\sigma_{1}\right]_{3}
$$

Concerning the number of elements $\sigma^{(n)} \in\{-1,1\}^{3 n+3}$ corresponding to $\left(s_{1}\right)-\left(s_{7}\right)$ we observe

Proposition 4.14.

For $n \geq 3$ there exist $(n-1)(n+2)$ elements in $\{-1,1\}^{3 \times(n+1)}$ of the form $\left(s_{1}\right)-\left(s_{7}\right)$.

Proof. We note that for $n \geq 3$ fixed formulae $\left(s_{5}\right)$ and $\left(s_{6}\right)$ each yield as many elements as there are partitions of $n$ into three positive integers (taking order into account). Similarly $\left(s_{1}\right),\left(s_{2}\right)$ and $\left.s_{4}\right)$ each yield as many elements as there are partitions of $n$ into two positive integers (taking order into account), while $\left(s_{7}\right)$ yields as many elements as there are partitions of $n-1$ into two positive integers (taking order into account). Finally, $s_{3}$ yields one element.

First we count the number of ways an integer can be partitioned into the sum of two positive integers (if order is taken into account). One has

$$
n=(n-1)+1=(n-2)+2=\cdots=(n-(n-1))+(n-1) .
$$


Note that there is no other way to partition $n$ into the sum of two positive integers. Hence we conclude that there is a total of $n-1$ ways to partition $n$ into two positive integers.

Second we count the number of partitions of $n$ into three positive integers. To this end we observe:

$$
\begin{aligned}
n & =(n-2)+(1+1) \\
& =(n-3)+\{2 \text { ways to write } 3 \text { as the sum of two positive integers }\} \\
& \vdots \\
& =(n-j)+\{j-1 \text { ways to write } j \text { as the sum of two positive integers }\} \\
& \vdots \\
& =(n-(n-1))+\{n-2 \text { ways to write } n-1 \text { as the sum of two positive integers }\}
\end{aligned}
$$

Consequently there is a total of

$$
\sum_{j=2}^{n-1}(j-1)=\frac{1}{2}(n-2)(n-1)
$$

ways to partition $n$ into the sum of three positive integers. Now we can sum over the contribution of each of the formulae

$$
\underbrace{(n-2)(n-1)}_{\left.s s_{5}\right)}+\underbrace{3(n-1)}_{s s_{6}}+\underbrace{3(n-2)}_{\left.s s_{1}\right]}+\underbrace{(1)}_{s s_{3}}=(n-1)(n+2) .
$$

\section{Proof of Theorem 4.2}

To prove the Theorem 4.2 we show the following implications:

$$
(A) \Rightarrow(D) \Rightarrow(C) \Rightarrow(B) \Rightarrow(A)
$$

$(A) \Rightarrow(D):$

Suppose there exists $a, b \in \mathbb{R}_{>0}^{3 n+3}$ and $\kappa \in \mathbb{R}_{>0}^{3 n+3}$ such that $14 \mathrm{a}$ \& $14 \mathrm{~b}$ hold. Then, by Theorem 4.3 of [20] one has

$$
\ln b-\ln a \in \operatorname{im}\left(A^{T}\right),
$$

with

$$
A=\left[\begin{array}{lll|lll|lll|l|lll}
1 & 0 & 0 & 1 & 1 & 1 & 2 & 2 & 2 & \ldots & n & n & n \\
1 & 0 & 1 & 1 & 0 & 1 & 1 & 0 & 1 & \ldots & 1 & 0 & 1 \\
0 & 1 & 0 & 1 & 1 & 1 & 1 & 1 & 1 & \ldots & 1 & 1 & 1
\end{array}\right],
$$

where the columns of $A$ have been reordered to match the ordering introduced in Table 1 (in the aforementioned reference [20], the columns of $A$ correspond to the exponents of $t_{1}, t_{2}, t_{3}$ in the statement of Theorem 4.3, the matrix itself is displayed in the proof of said Theorem).

$$
A^{T}=M^{(n)} Q \text { for the nonsingular } Q:=\left[\begin{array}{ccc}
n & 0 & 1 \\
1 & 1 & 1 \\
2 & 1 & 1
\end{array}\right]
$$


implying $\operatorname{im}\left(M^{(n)}\right)=\operatorname{im}\left(A^{T}\right)$ and hence $(A) \Rightarrow(D)$ with $\mu:=\ln b-\ln a$.

$(D) \Rightarrow(C)$ :

We first show that $\mathcal{Y}^{(n)^{T}} M^{(n)}=\Pi^{(n)}$. To this end recall the definition of $\mathcal{Y}^{(n)^{T}}$ from eq.9 $9 \mathrm{~d}$

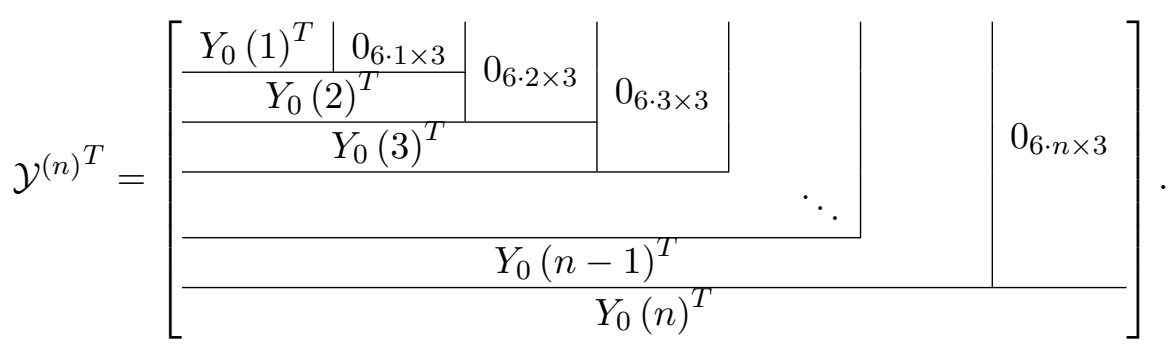

Now it is straightforward to compute that

$$
Y_{0}(i)^{T}\left[\begin{array}{c}
M(0, n) \\
\vdots \\
M(i, n)
\end{array}\right]=\left[\begin{array}{lll}
\underline{0} & (-i+2) \underline{1} & (i-1) \underline{1}
\end{array}\right]=: P(i) \in \mathbb{R}^{6 \times 3},
$$

where, as in Definition 4.1 we use the symbol 1 to denote a 6 -vector filled with the number 1 and, accordingly, we use the symbol $\underline{0}$ to denote a 6 -vector filled with the number 0 . Thus one arrives at

$$
\mathcal{Y}^{(n)^{T}} M^{(n)}=\left[\begin{array}{c}
P(1) \\
\vdots \\
P(n)
\end{array}\right]
$$

Further note that

$$
P(i)\left(\begin{array}{l}
* \\
\xi_{1} \\
\xi_{2}
\end{array}\right)=\left[\begin{array}{lll}
(-i+2) \underline{1} & (i-1) \underline{1}
\end{array}\right]\left(\begin{array}{l}
\xi_{1} \\
\xi_{2}
\end{array}\right)=\Pi_{0}(i)\left(\begin{array}{l}
\xi_{1} \\
\xi_{2}
\end{array}\right)
$$

(cf. Definition 4.1 and in particular equation $16 \mathrm{a})$ ). Thus, given any nonzero $\mu \in \operatorname{im}\left(M^{(n)}\right)$ as

$$
\mu=M^{(n)}\left(\begin{array}{l}
* \\
\xi_{1} \\
\xi_{2}
\end{array}\right)
$$

one arrives at

$$
\mathcal{Y}^{(n)^{T}} M^{(n)}\left(\begin{array}{l}
* \\
\xi_{1} \\
\xi_{2}
\end{array}\right)=\left[\begin{array}{c}
\Pi_{0}(1) \\
\vdots \\
\Pi_{0}(n)
\end{array}\right]\left(\begin{array}{l}
\xi_{1} \\
\xi_{2}
\end{array}\right)=\Pi^{(n)} \xi .
$$

Concerning $\nu$ and $\lambda$ as in $(19 \mathrm{~b}) \&(19 \mathrm{c})-(19 \mathrm{e})$ we note that $\xi_{1}, \xi_{2}$ from above together with any positive $\lambda$ can be used to obtain $\nu$ as in $(19 \mathrm{c})-(19 \mathrm{e})$. Hence we conclude $(D) \Rightarrow(C)$.

$(C) \Rightarrow(B)$ :

Let $\mu \in \mathbb{R}^{3 n+3}$ with $\mu \neq 0$ and $\xi \in \mathbb{R}^{2}$ be given, such that 19 a holds. Further let $\nu$ and $\lambda$ be given that satisfy $(19 \mathrm{~b}) \&(19 \mathrm{c})-(19 \mathrm{e})$, that is $\lambda \in \mathbb{R}_{>0}^{3 n}$ and

$$
\nu_{3 i}=\lambda_{3 i} e^{(2-i) \xi_{1}+(i-1) \xi_{2}}, \nu_{3 i-1}=\lambda_{3 i-1} \frac{\nu_{3 i}}{\lambda_{3 i}} \text { and } \nu_{3 i-2}=\lambda_{3 i-2} \frac{\nu_{3 i}}{\lambda_{3 i}}, i=1, \ldots, n \text {. }
$$

Observe that these $\nu, \lambda$ satisfy

and hence

$$
\ln \frac{\nu_{3 i-2}}{\lambda_{3 i-2}}=\ln \frac{\nu_{3 i-1}}{\lambda_{3 i-1}}=\ln \frac{\nu_{3 i}}{\lambda_{3 i}}=(2-i) \xi_{1}+(i-1) \xi_{2}
$$

$$
\ln \frac{\nu_{3 i-2}+\nu_{3 i}}{\lambda_{3 i-2}+\lambda_{3 i}}=\ln \frac{\nu_{3 i}}{\lambda_{3 i}} \text { and } \ln \frac{\nu_{3 i-1}+\nu_{3 i}}{\lambda_{3 i-1}+\lambda_{3 i}}=\ln \frac{\nu_{3 i}}{\lambda_{3 i}} .
$$


Define

$$
\nu_{(i)}:=\left(\nu_{3 i-2}, \nu_{3 i-1}, \nu_{3 i}\right) \text { and } \lambda_{(i)}:=\left(\lambda_{3 i-2}, \lambda_{3 i-1}, \lambda_{3 i}\right)
$$

and let

$$
\nu=\operatorname{col}\left(\nu_{(1)}, \ldots, \nu_{(n)}\right) \text { and } \operatorname{col}\left(\lambda_{(1)}, \ldots, \lambda_{(n)}\right) .
$$

Recall the matrices $E^{(n)}$ and $E$ from eqns. $\left.15 \mathrm{~b}\right) \&(15 \mathrm{a})$ and observe that

$$
\ln \frac{E^{(n)} \nu}{E^{(n)} \lambda}=\left(\ln \frac{E \nu_{(1)}}{E \lambda_{(1)}}, \ldots, \ln \frac{E \nu_{(n)}}{E \lambda_{(n)}}\right)
$$

and

$$
\ln \frac{E \nu_{(i)}}{E \lambda_{(i)}}=\left(\ln \frac{\nu_{3 i-2}+\nu_{3 i}}{\lambda_{3 i-2}+\lambda_{3 i}}, \ln \frac{\nu_{3 i-2}}{\lambda_{3 i_{2}}}, \ln \frac{\nu_{3 i}}{\lambda_{3 i}}, \ln \frac{\nu_{3 i-1}+\nu_{3 i}}{\lambda_{3 i-1}+\lambda_{3 i}}, \ln \frac{\nu_{3 i-1}}{\lambda_{3 i-1}}, \ln \frac{\nu_{3 i}}{\lambda_{3 i}}\right) .
$$

Hence one has for $\nu, \lambda$ from above

$$
\ln \frac{E \nu_{(i)}}{E \lambda_{(i)}}=\left[\begin{array}{lll}
(2-i) & \underline{1} & (i-1)
\end{array}\right]\left(\begin{array}{l}
\xi_{1} \\
\xi_{2}
\end{array}\right)
$$

and

$$
\ln \frac{E^{(n)} \nu}{E^{(n)} \lambda}=\Pi^{(n)} \xi
$$

Consequently, if there exist vectors $\mu \in \mathbb{R}^{3 n+3}, \mu \neq 0$ and $\xi \in \mathbb{R}^{2}$ satisfying (19a) and vectors $\nu, \lambda$ satisfying (19b), (19c) - 19e), then $\mu, \nu$ and $\lambda$ also satisfy (18). Note that $\nu$ and $\lambda$ satisfying (19b) $\& \sqrt{19 \mathrm{c})}-(19 \mathrm{e})$ are positive. Thus we conclude $(C) \Rightarrow(B)$.

$(B) \Rightarrow(A)$ :

Finally, assume $\mu \in \mathbb{R}^{3 n+3}$ with $\mu \neq 0$ and $(\nu, \lambda) \in \mathbb{R}_{>0}^{3 n} \times \mathbb{R}_{>0}^{3 n}$ satisfy $(18)$. Fix $a \in \mathbb{R}_{>0}^{n}$ and define

$$
\begin{aligned}
b & :=\operatorname{diag}\left(e^{\mu}\right) a \\
\kappa & :=\operatorname{diag}\left(\Phi^{(n)}\left(a^{-1}\right)\right) E^{(n)} \lambda .
\end{aligned}
$$

Then

$$
S^{(n)} \operatorname{diag}(\kappa) \Phi^{(n)}(a)=S^{(n)} \operatorname{diag}\left(\Phi^{(n)}\left(a^{-1}\right)\right) \operatorname{diag}\left(E^{(n)} \lambda\right) \Phi^{(n)}(a)=S^{(n)} E^{(n)} \lambda=0
$$

and (as, by $\left.18, \mathcal{Y}^{(n)^{T}} \mu=\ln \frac{E^{(n)} \nu}{E^{(n)} \lambda}\right)$

$$
S^{(n)} \operatorname{diag}(\kappa) \Phi^{(n)}(b)=S^{(n)} \operatorname{diag}(\kappa) \operatorname{diag}\left(\Phi^{(n)}(a)\right) e^{\mathcal{Y}^{(n)^{T}} \mu}=S^{(n)} E^{(n)} \nu=0
$$

and therefore $(B) \Rightarrow(A)$ where we have used equation $13 \mathrm{a}$ of Remark 3.4 .

\section{Proof of Theorem 4.10}

First, we observe that $\operatorname{sign}\left(M^{(n)} \xi\right)=\sigma^{(n)}$ implies $\operatorname{sign}\left(M^{(n)}(-\xi)\right)=-\sigma^{(n)}$ and that $Z^{(n)} s=0$ implies $Z^{(n)}(-s)=0$. Hence, if the system 28$)$ associated to $\sigma^{(n)}$ is feasible for $(\xi, s)$, then the system 28 associated to $-\sigma^{(n)}$ is feasible for $(-\xi,-s)$. Thus $\sigma^{(n)} \in \mathcal{M}^{(n)}$ implies $-\sigma^{(n)} \in \mathcal{M}^{(n)}$. 
Hence it suffices to show that the linear system 28 is feasible for elements $\sigma^{(n)} \in\{-1,1\}^{3 n+3}$ with $\sigma_{3 n}=+1$ if and only if $\sigma^{(n)}$ corresponds to one of the sign pattern matrices in $\left.\left(s_{1}\right)-s_{7}\right)$.

We now turn to the necessity of the sign patterns $\left(s_{1}\right)-\left(s_{7}\right)$. To this end we parametrize the range of the regular matrix $M(0, n)$ from eq. (17a) by

$$
w(0):=\left[\begin{array}{c}
y \\
x \\
y-z
\end{array}\right]=\left[\begin{array}{ccc}
-1 & -n+1 & n \\
1 & n & -n \\
-1 & -n+2 & n-1
\end{array}\right]\left[\begin{array}{c}
x+n z \\
x+y \\
x+y+z
\end{array}\right] .
$$

Consequently, one has for $M(i, n), i=1, \ldots, n$, from eq. $17 \mathrm{~b}$

$$
w(i)=\left[\begin{array}{l}
w_{1}(i) \\
w_{2}(i) \\
w_{3}(i)
\end{array}\right]:=\left[\begin{array}{c}
x+i z+y-z \\
x+i z \\
x+i z+y-z
\end{array}\right]=\left[\begin{array}{rrr}
0 & -i+2 & i-1 \\
1 & n-i & -n+i \\
0 & -i+2 & i-1
\end{array}\right]\left[\begin{array}{c}
x+n z \\
x+y \\
x+y+z
\end{array}\right] .
$$

Observe that the components $w_{2}(i)$ and $w_{1}(i)=w_{3}(i)=w_{2}(i)+(y-z)$ are affine functions of $i$ so sign changes can be easily read off. We now derive the necessary conditions for sign $(w(0))$ to be one of the four sign patterns $\sigma_{1}, \sigma_{2}, \sigma_{4}$ and $\sigma_{7}$ from (33a) - 33c under the side condition that system (31) is not unfeasible (cf. Lemma 4.9). The indices $i_{k}$ and $j_{k}$ to follow will always be $\geq 1$.

(1) $\operatorname{sign}(w(0))=\sigma_{2}$, i.e., $y>0, x<0, y-z>0$ :

For $z \leq 0$, the $w_{2 i}$ are all negative so that one has $\sigma_{20}=\sigma_{21}=\cdots=\sigma_{2 n}=-1$ and $\sigma_{10}=\sigma_{30}=1$. By item (2) of Lemma 4.9, $z \leq 0$ cannot lead to a feasible sign pattern. For $z>0$ the possible sign pattern matrices are

$$
\left(\sigma_{2},\left[\sigma_{3}\right]_{i_{1}},\left[\sigma_{1}\right]_{i_{2}}\right), \quad\left(\sigma_{2},\left[\sigma_{3}\right]_{j_{1}},\left[\sigma_{2}\right]_{j_{2}},\left[\sigma_{1}\right]_{j_{3}}\right)
$$

for $i_{1}+i_{2}=n$ and $j_{1}+j_{2}+j_{3}=n$ where the $\sigma_{1}$ entries are necessary by item (2) of Lemma 4.9 . Observe that the sign pattern $\left(\sigma_{2},\left[\sigma_{1}\right]_{n}\right)$ is unfeasible by item (1) of Lemma 4.9.

(2) $\operatorname{sign}(w(0))=\sigma_{3}$, i.e., $y<0, x<0, y-z<0$ :

For $z \leq 0$, the components of all the $w(i)$ are of the same sign. By item (1) of Lemma 4.9. $z \leq 0$ cannot lead to a feasible sign pattern. For $z>0$, the $w(i)$ may generate the sign pattern matrices

$$
\left(\sigma_{3},\left[\sigma_{5}\right]_{i_{1}},\left[\sigma_{1}\right]_{i_{2}}\right), \quad\left(\sigma_{3},\left[\sigma_{3}\right]_{j_{1}},\left[\sigma_{5}\right]_{j_{2}},\left[\sigma_{1}\right]_{j_{3}}\right)
$$

with $i_{1}+i_{2}=n$ and $j_{1}+j_{2}+j_{3}=n$ where the $\sigma_{1}$ entries are necessary (otherwise the first rows do not offer a sign change). The direct passage from $\sigma_{3}$ to $\sigma_{1}$ is obviously impossible since $w_{2}(i)<0$ implies $w_{1}(i+1)=w_{2}(i)+y \leq w_{2}(i)<0$.

(3) $\operatorname{sign}(w(0))=\sigma_{4}$, i.e., $x<0,0<y<z$ :

At first, possible sign pattern matrices are

$$
\left(\sigma_{4},\left[\sigma_{3}\right]_{i_{1}},\left[\sigma_{1}\right]_{i_{2}}\right), \quad\left(\sigma_{4},\left[\sigma_{5}\right]_{i_{1}},\left[\sigma_{1}\right]_{i_{2}}\right), \quad\left(\sigma_{4},\left[\sigma_{3}\right]_{j_{1}},\left[\sigma_{5}\right]_{j_{2}},\left[\sigma_{1}\right]_{j_{3}}\right)
$$

for $i_{1}+i_{2}=n$ and $j_{1}+j_{2}+j_{3}=n$ where the $\sigma_{1}$ entries are necessary by item (3) of Lemma 4.9 . Observe that the sign pattern $\left(\sigma_{4},\left[\sigma_{1}\right]_{n}\right)$ is unfeasible by item (1) of Lemma 4.9. Note that $\sigma_{5}$ can appear just once because of $w_{1, i+1}=w_{2 i}+y \geq w_{2 i}$.

(4) $\operatorname{sign}(w(0))=\sigma_{7}$, i.e., $x>0,0<y<z$ :

Because of $z>0$, the components $w_{1 i}$ are positive for $i=0, \ldots, n$. By item (1) of Lemma 4.9. $\sigma_{7}$ cannot lead to a feasible sign pattern.

We finally establish the sufficiency of the sign patterns $\left(s_{1}\right)-\left(s_{7}\right)$. By Lemma 4.9 , these $\left(s_{k}\right)$ are feasible sign patterns for the kernel of $Z^{(n)}$. In order to prove that they are realizable we present for each $\left(s_{k}\right), k \in\{1, \ldots, 7\}$, a vector

$$
\xi=\operatorname{col}(x, x+y, x+y+z) \in \mathbb{R}^{3}
$$


leading to the sign pattern $\left(s_{k}\right)$ for $M^{(n)} \xi$ :

$$
\begin{array}{llll}
\text { For }\left(s_{1}\right): & x=i_{2}-\frac{3}{4}, & y=-n+\frac{5}{4}, & z=1 . \\
\text { For }\left(s_{2}\right): & x=n-\frac{1}{4}, & y=-n-i_{1}+\frac{3}{4}, & z=1 . \\
\text { For }\left(s_{3}\right): & x=n-\frac{3}{4}, & y=-n+\frac{1}{4}, & z=1 . \\
\text { For }\left(s_{4}\right): & x=i_{2}-\frac{1}{4}, & y=-n+\frac{3}{4}, & z=1 . \\
\text { For }\left(s_{5}\right): & x=i_{3}-\frac{3}{4}, & y=-n+i_{2}+\frac{5}{4}, & z=1 . \\
\text { For }\left(s_{6}\right): & x=i_{2}+i_{3}-\frac{1}{4}, & y=-n-i_{2}+\frac{3}{4}, & z=1 . \\
\text { For }\left(s_{7}\right): & x=i_{2}+\frac{1}{4}, & y=-n+\frac{1}{4}, & z=1 .
\end{array}
$$

\section{Discussion}

The main topic of this contribution is the existence of multistationarity for $n$-site sequential distributive phosphorylation. Via the equations $21 \mathrm{c}$ ) and $27 \mathrm{a})-(27 \mathrm{c})$ we were able to link the existence of pairs of steady states $(a, b)$ and a corresponding vector of rate constants $\kappa$ to solutions of the linear systems 28). These systems are uniquely defined by sign patterns $\sigma^{(n)} \in\{-1,0,1\}^{3 n+3}$ and hence the existence of a single sign pattern defining a feasible system (28) is sufficient. Concerning sign patterns $\sigma \in\{-1,1\}^{3 n+3}$ where $(28)$ is feasible, Theorem 4.10 establishes four such sign patterns for $n=2$ and $(n-1)(n+2)$ for $n \geq 3$.

Unfortunately the biological interpretation of elements $\sigma$ where (28) is feasible is unclear (unlike in the case of multistationarity itself, that is usually associated with a specific biological function). If different elements could be associated to different biological functions, this would limit the number of (different) biological functions a network can perform and hence be of significant biological interest. Moreover, knowledge of all sign patterns $\sigma$ defining feasible (28) would then correspond to knowledge of all biological functions a network is capable of.

However, the following observations concerning pairs of steady states $(a, b)$ can easily be made by inspection of the feasible sign patterns $\left(s_{1}\right)-\left(s_{7}\right)$ and the structure of the matrices $M^{(i)}$ from 17b): (i) every sign pattern ends with the triplet $(1,1,1)^{T}$ or $(-1,-1,-1)^{T}$ and hence the last three components of steady state $b$ are greater (smaller) than those of $a$ (recall that $\mu=\ln \frac{b_{i}}{a_{i}}$ and hence $\mu_{i}>0$ implies $b_{i}>a_{i}$ and $\mu_{i}<0$ implies $b_{i}<a_{i}$ ). (ii) The first and the third row of the matrices $M^{(i)}$ are identical. Hence $\mu_{3 i+1}=\mu_{3 i+3}, i=1, \ldots, n$. For a pair of steady states $(a, b)$ these correspond to the ratio of steady state concentrations of kinase-substrate complex $A_{i-1 p} E_{1}$ (in case of $\mu_{3 i+1}$ ) and phosphatase-substrate complex $A_{i_{p}} E_{2}$ (in case of $\mu_{3 i+3}$, cf. Table 1). Hence, for any pair of steady states, the ratio of the steady state concentrations of kinase-substrate complexes equals that of phosphatase-substrate complexes.

Finally we'd like to discuss two observations related to the formulae $(21 \mathrm{c})$ and $(27 \mathrm{a})-(27 \mathrm{c})$. Consider a sign pattern matrix $\sigma$ as in $\left(s_{1}-s_{7}\right)$ and let $\sigma^{(n)}$ be the corresponding sign pattern vector. Pick vectors $\mu \in \operatorname{im}\left(M^{(n)}\right), s \in \operatorname{im}\left(S^{(n)}\right)$ with $\operatorname{sign}(s)=\operatorname{sign}(\mu)=\sigma^{(n)}$. Let $\left(a^{\sigma}, b^{\sigma}\right)$ be the pair of steady states defined by $\mu, s$ via $27 \mathrm{a})-(27 \mathrm{c})$. Let $\left(a^{-\sigma}, b^{-\sigma}\right)$ be the pair of steady states defined by $-\mu$ and $-s$. Then it is not difficult to see that $a^{-\sigma}=b^{\sigma}$ and $b^{-\sigma}=a^{\sigma}$ (cf. a similar discussion in [4]). Further note that a given pair $(a, b)$, obtained via $(27 a)-(27 c)$, is a pair of steady states for all vectors of rate constants defined by the formula $21 \mathrm{c})$, where $\lambda \in \mathbb{R}_{>0}^{3 n}$ is free.

The formula $(21 \mathrm{c})$ for the vectors of rate constants may be interesting for two reasons: first, it allows to replace the $6 n$ rate constants $k_{i}, l_{i}$ by the $3 n$ 'coordinates' $\lambda_{i}$ and hence a reduction of the degrees of freedom in the dynamical system (12). Second, it means that multistationarity is robust with respect to perturbations within the image of the map (21c) and fragile with respect to transversal perturbations. In a future publication we will explore the image of this map with the aim of establishing parameter values for multistationarity in biologically meaningful domains. 
7.1. Acknowledgments. $\mathrm{KH}$ and $\mathrm{CC}$ acknowledge financial support from the International Max Planck Research School in Magdeburg and the Research Center 'Dynamic Systems' of the Ministry of Education of Saxony-Anhalt, respectively. Finally, we'd like to thank the diligent reviewers for their valuable suggestions.

\section{REFERENCES}

[1] D. Barik, W. T. Baumann, M. R. Paul, B. Novak, and J. J. Tyson. A model of yeast cell-cycle regulation based on multisite phosphorylation. Molecular Systems Biology, 6:1-18, 2010.

[2] K. C. Chen, L. Calzone, A. Csikasz-Nagy, F. R. Cross, B. Novak, and J. J. Tyson. Integrative analysis of cell cycle control in budding yeast. Molecular Biology of the Cell, 15(8):3841-3862, 2004.

[3] C. Conradi and D. Flockerzi. Multistationarity in mass action networks with applications to ERK activation. Journal of Mathematical Biology, 65(1):107-156, 2012.

[4] C. Conradi, D. Flockerzi, and J. Raisch. Multistationarity in the activation of a MAPK: Parametrizing the relevant region in parameter space. Mathematical Biosciences, 211:05-131, 2008.

[5] C. Conradi, J. Saez-Rodriguez, E.-D. Gilles, and J. Raisch. Using chemical reaction network theory to discard a kinetic mechanism hypothesis. Systems Biology, IEE Proceedings (now IET Systems Biology), 152(4):243-248, 2005.

[6] C. Conradi, J. Saez-Rodriguez, E.-D. Gilles, and J. Raisch. Chemical Reaction Network Theory - a tool for systems biology. Proceedings of the 5th MATHMOD, 2006.

[7] M. T. Cooling, P. Hunter, and E. J. Crampin. Sensitivity of NFAT cycling to cytosolic calcium concentration: implications for hypertrophic signals in cardiac myocytes. Biophysical Journal, 96:2095-2104, 2009.

[8] G. R. Crabtree and E. N. Olson. NFAT signaling: Choreographing the social lives of cells. Cell, 109(2, Supplement 1):S67-S79, 2002.

[9] M. Feinberg. The existence and uniqueness of steady states for a class of chemical reaction networks. Archive for Rational Mechanics and Analysis, 132:311-370, 1995.

[10] M. Feinberg. Multiple steady states for chemical reaction networks of deficiency one. Archive for Rational Mechanics and Analysis, 132:371-406, 1995.

[11] E. Feliu and C. Wiuf. Enzyme-sharing as a cause of multi-stationarity in signalling systems. Journal of The Royal Society Interface, 9(71):1224-1232, 2012.

[12] J. Gunawardena. Multisite protein phosphorylation makes a good threshold but can be a poor switch. Proceedings of the National Academy of Sciences, 102(41):14617-14622, 2005.

[13] J. Gunawardena. Distributivity and processivity in multisite phosphorylation can be distinguished through steady-state invariants. Biophysical Journal, 93(11):3828-3834, 2007.

[14] N. Hermann-Kleiter and G. Baier. NFAT pulls the strings during CD4+ T helper cell effector functions. Blood, 115(15):2989-2997, 2010.

[15] K. Holstein, D. Flockerzi, and C. Conradi. Parameter regimes for bistability in multisite phosphorylation networks. in preparation, 2013.

[16] C.-Y. F. Huang and J. E. Ferrell. Ultrasensitivity in the mitogen-activated protein kinase cascade. Proceedings of the National Academy of Science, 93(19):10078-10083, 1996.

[17] S. Klamt and J. Gagneur. Computation of elementary modes: a unifying framework and the new binary approach. BMC Bioninformatics, 175(5), 2004.

[18] F. Macian. NFAT proteins: key regulators of T-cell development and function. Nature Reviews Immunology, $5(6): 472-484,2005$.

[19] N. I. Markevich, J. B. Hoek, and B. N. Kholodenko. Signaling switches and bistability arising from multisite phosphorylation in protein kinase cascades. Journal of Cell Biology, 164(3):353-359, 2004.

[20] M. Pérez Millán, A. Dickenstein, A. Shiu, and C. Conradi. Chemical reaction systems with toric steady states. Bulletin of Mathematical Biology, 74:1027-1065, 2012.

[21] C. Salazar and T. Höfer. Versatile regulation of multisite protein phosphorylation by order of phosphate processing and protein-protein interactions. Federation of European Biochemical Societies, 274:1046-1061, 2007.

[22] C. Salazar and T. Höfer. Multisite protein phosphorylation - from molecular mechanisms to kinetic models. FEBS Journal, 276(12):3177-3198, 2009.

[23] R. Seger and E. G. Krebs. The MAPK signaling cascade. The FASEB Journal, 9:726-735, 1995.

[24] Y. D. Shaul and R. Seger. The MEK/ERK cascade: From signaling specificity to diverse functions. Biochimica et Biophysica Acta (BBA) - Molecular Cell Research, 1773(8):1213-1226, 2007.

[25] M. Thomson and J. Gunawardena. The rational parameterisation theorem for multisite post-translational modification systems. Journal of Theoretical Biology, 261(4):626-636, 2009.

[26] M. Thomson and J. Gunawardena. Unlimited multistability in multisite phosphorylation systems. Nature, 460(7252):274-277, 2009. 
[27] T. Tomida, K. Hirose, A. Takizawa, F. Shibasaki, and M. Iino. NFAT functions as a working memory of $\mathrm{Ca}^{+2}$ signals in decoding $\mathrm{Ca}^{+2}$ oscillation. The European Molecular Biology Organization Journal, 22(15):3825-3832, 2003.

[28] J. J. Tyson and B. Novák. Temporal organization of the cell cycle. Current Biology, 18:R759-R768, 2008.

[29] M. Uhr. Structural analysis of inference problems arising in systems biology. PhD thesis, ETH Zurich, 2012.

[30] L. Wang and E. Sontag. On the number of steady states in a multiple futile cycle. Journal of Mathematical Biology, 57:29-52, 2008.

[31] Z. Yao and R. Seger. The ERK signaling cascade - views from different subcellular compartments. BioFactors, 35(5):407-416, 2009.

Max-Planck-Institut Dynamik komplexer technischer Systeme, Sandtorstr. 1, 39106 Magdeburg, GERMANY.

E-mail address: holstein@mpi-magdeburg.mpg.de,flockerzi@mpi-magdeburg.mpg.de, conradi@mpi-magdeburg.mpg.de 